\title{
Gaussoids are two-antecedental approximations of Gaussian conditional independence structures
}

\author{
Tobias Boege ${ }^{1}$ (D)
}

Accepted: 3 November 2021/Published online: 25 November 2021

(C) The Author(s) 2022

\begin{abstract}
The gaussoid axioms are conditional independence inference rules which characterize regular Gaussian CI structures over a three-element ground set. It is known that no finite set of inference rules completely describes regular Gaussian CI as the ground set grows. In this article we show that the gaussoid axioms logically imply every inference rule of at most two antecedents which is valid for regular Gaussians over any ground set. The proof is accomplished by exhibiting for each inclusion-minimal gaussoid extension of at most two CI statements a regular Gaussian realization. Moreover we prove that all those gaussoids have rational positive-definite realizations inside every $\varepsilon$-ball around the identity matrix. For the proof we introduce the concept of algebraic Gaussians over arbitrary fields and of positive Gaussians over ordered fields and obtain the same two-antecedental completeness of the gaussoid axioms for algebraic and positive Gaussians over all fields of characteristic zero as a byproduct.
\end{abstract}

Keywords Conditional independence · Inference · Completeness · Gaussoid · Realizability · Rationality

Mathematics Subject Classification (2010) 62B10 · 62R01 · 14P10

\section{Introduction}

Conditional independence $(\mathrm{CI})$ is a basic notion in the probabilistic approach to reasoning under uncertainty. CI constraints prescribe statements of the form "given that the value of the factor $C$ is known, the value of $A$ is irrelevant to the value of $B$ ", or "having observed $C$, the outcome of $A$ gives no further information on the outcome of $B$ " on a joint probability distribution of random variables $A, B, C, \ldots$ Such statements allow the incorporation of expert knowledge on independences of the real-world phenomena into a statistical model. The importance of conditional independence in statistical theory has been described in a

Tobias Boege

tobias.boege@mis.mpg.de

1 Max-Planck-Institut für Mathematik in den Naturwissenschaften, Inselstraße 22, 04103,

Leipzig, Germany 
seminal paper by Dawid [7]. Pearl [28, Chapter 3] further emphasizes the usefulness of CI as a qualitative, as opposed to numeric, measure of independence in artificial intelligence, in that reasoning on CI can be performed logically instead of by summing over and dividing given probabilities. The advantage of using CI inference in the processing and deduction of further (in)dependence statements is that every conclusion which is drawn from a given set of $\mathrm{CI}$ assumptions is sound and that their derivation happens in discrete steps, each of which is verifiable by humans based on agreed-upon deduction rules.

The fundamental laws of conditional independence identified by Dawid became the definition of semigraphoids. The semigraphoid properties are universal deduction rules which are valid for all probability distributions [33, Lemma 2.1]. Thus they may immediately be applied to any set of statements about the conditional independences among random variables to derive additional knowledge about the independence structure of the stochastic system. The historical reference on the topic, after Dawid, is [29], where the special case of graphoids is introduced and first named in the context of graphical models. See also the historical overview in [34]. By deduction or inference rules, we always mean CI inference formulas (or inference forms)

$$
\bigwedge_{s} a_{s} \Rightarrow \bigvee_{t} c_{t}
$$

demanding that if all of the antecedent statements $a_{s}$ are satisfied, then at least one of the consequent statements $c_{t}$ is satisfied. Which inference formulas are true, i.e., inference rules, depends on assumptions about the type of probability distribution. Distributions with only binary random variables may satisfy more inference rules than arbitrary discrete distributions, which may satisfy yet other rules than the continuous Gaussian distributions. For this reason, the structures of conditional independence for restricted classes of distributions have become objects of mathematical study in their own right. Given a class of distributions, such as Gaussians, a central task is to solve the inference problem: to decide when an inference form $(\Rightarrow)$ is valid for all distributions in the class, and then to list all of them for use in expert systems. See [10] for early work and the recent survey [1].

For a detailed introduction to probabilistic representations of CI structures, we refer to [33]. The recent book [38] contains an algebraically-minded introduction to CI of discrete and Gaussian distributions in Chapter 4. Chapter 13 of the same book discusses undirected and directed acyclic graphical models. These have been of interest since at least the works of Lauritzen [15] and Pearl [28] and have found many uses in practice. These types of graphs are special cases of both, discrete as well as Gaussian, CI models; see [38, Section 13.2]. An overview and unification of the CI inference calculi of various other sorts of graphical models can be found in [16].

The research into CI structures of discrete random variables in the late 1980s was in part driven by the conjecture of Pearl and Paz that to every semigraphoid a matching vector of discrete random variables may be found whose CI structure is exactly that semigraphoid [28, p. 88]. An alternative formulation of this conjecture, in logical terminology, is that the semigraphoid axioms are complete for the theory of discrete CI structures. This conjecture was refuted by Studený who exhibited an infinite family of valid inference rules for discrete CI which are not implied by any finite set of valid inference rules [35]. This not only proves that the semigraphoid axioms are not complete, but that no finite list of axioms can be complete for discrete CI. Studený's inference rules naturally require a growing number of random variables but they also use more and more antecedents. A finite complete list of valid inference rules being impossible to obtain, the conjecture was revised to state that the semigraphoid axioms are complete for only those inference rules of discrete CI which 
have at most two antecedents, as the semigraphoid axioms themselves do. This was in turn resolved positively by Studený [36]. In this sense, the semigraphoid axioms are the most fundamental laws of $\mathrm{CI}$ on discrete random vectors: they logically imply all the non-trivial valid inference rules with the lowest number of antecedents, or premises - those which one would expect to be most acceptable in human reasoning.

The same story unfolds for multivariate regular Gaussian distributions, with more or less 15 years delay. In the case of Gaussian distributions, CI structures strike a fine balance between varied and highly structured. For example, the inference mechanisms of Markov and Bayesian networks in graphical modeling are special cases of Gaussian CI models, but the general theory is not more complicated than real algebraic geometry (see Remark 4.1). The role of the semigraphoid axioms as the most essential inference rules is here played by the gaussoid axioms. These, likewise two-antecedental, inference rules have been found by Matúš in [22] and the term gaussoid was coined in the work [17] by Lněnička and Matúš who classified the CI structures which arise from Gaussians on three and four random variables. Realizability in the three-variate case is characterized by the gaussoid axioms. On four random variables, higher inference rules arise, but none with only two antecedents. Around the same time, Sullivant [37], and independently Šimeček [30] in a different framework, showed that no finite list of inference rules suffices to deduce all valid inference rules for $n$-variate Gaussian distributions, as $n$ grows. In this article we prove the Gaussian analogue of Studený's two-antecedental completeness result in Corollary 3.1: every valid inference rule for Gaussians on any number of random variables but with at most two antecedents can be deduced from the gaussoid axioms. Hence, the gaussoid axioms are the most essential deduction rules for CI inference on Gaussian random variables.

This work is structured as follows: Section 2 gives an introduction to Gaussian conditional independence structures and their symmetries. Section 3 gives a precise statement of the main technical result Theorem 3.1 and derives the titular two-antecedental completeness result Corollary 3.1 from it. At the end of Section 3, a more detailed summary of the proof strategy is given. The subsequent sections then form the technical part of the paper which execute the strategy: Section 4 discusses an algebraic relaxation of Gaussian distributions together with their symmetries. Section 5 shows how Gaussian realizability results can be recovered from this relaxation. The proof of Theorem 3.1 is presented in a series of lemmas in Section 6. Section 7 contains further examples and discussion of future work.

\section{Preliminaries}

We fix a finite ground set $N$ of size $n$ which labels a vector $\xi=\left(\xi_{i}\right)_{i \in N}$ of random variables. Suppose that this vector follows a multivariate Gaussian distribution with mean vector $\mu$ and positive-definite covariance matrix $\Sigma$. Its density with respect to the Lebesgue measure on $\mathbb{R}^{N}$ is given by

$$
x \mapsto \frac{1}{\sqrt{(2 \pi)^{n} \operatorname{det} \Sigma}} \exp \left(-\frac{1}{2}(x-\mu)^{T} \Sigma^{-1}(x-\mu)\right) .
$$

For distinct $i, j \in N$ and a disjoint subset $K \subseteq N$, the conditional independence statement $\xi_{i} \Perp \xi_{j} \mid \xi_{K}$ asserts, informally, that whenever the outcome of the subvector $\xi_{K}=\left(\xi_{k}\right)_{k \in K}$ is known, the outcomes of $\xi_{i}$ and $\xi_{j}$ are stochastically independent - learning one value provides no additional information on the other. The CI statement above is abbreviated by the symbol $(i j \mid K)$. In dealing with elements and subsets of the ground set $N$, we adopt the following notational conventions: (1) an element $i \in N$ may be written in place of the 
singleton subset $\{i\} \subseteq N$, and (2) juxtaposition $K L$ of subsets of $N$ abbreviates set union. In particular $i j K$ stands for $\{i\} \cup\{j\} \cup K \subseteq N$ and $i j=j i$ holds. This does not make the CI statement $(i j \mid K)$ ambiguous because conditional independence of random vectors is symmetric in $i$ and $j$. Let $\mathcal{A}_{N}:=\left\{(i j \mid K): i j \in\left(\begin{array}{c}N \\ 2\end{array}\right), K \subseteq N \backslash i j\right\}$ denote the set of all CI statements on $N$.

For a Gaussian vector $\xi$ with positive-definite covariance matrix $\Sigma$, conditional independence has an algebraic characterization (see [38, Proposition 4.1.9]):

$$
(i j \mid K) \text { holds for } \xi \Leftrightarrow \operatorname{det} \Sigma_{i K, j K}=0 \text {, }
$$

where $\Sigma_{i K, j K}$ is the submatrix of $\Sigma$ with rows $i K$ and columns $j K$. A submatrix of the form $\Sigma_{K, K}$ with equal row and column sets is principal. A symmetric matrix $\Sigma$ is positivedefinite if and only if all principal minors det $\Sigma_{K, K}, K \subseteq N$, are positive. In the submatrix $\Sigma_{i K, j K}$, which is decisive for the CI statement $(i j \mid K)$, the row and column sets $i K$ and $j K$ differ by only one element each. They are not principal but almost-principal submatrices. Hence, the true conditional independence statements of a given Gaussian distribution can be determined by evaluating the almost-principal minors of the covariance matrix. The mean $\mu$ plays no role and may be assumed to be zero. The CI structure of $\Sigma$ is the set of all valid CI statements:

$$
\llbracket \Sigma \rrbracket:=\left\{(i j \mid K) \in \mathcal{A}_{N}: \operatorname{det} \Sigma_{i K, j K}=0\right\}
$$

Example 2.1 Let $\left(\xi_{1}, \xi_{2}, \xi_{3}\right)$ follow a Gaussian distribution with zero mean and covariance matrix

$$
\Sigma=\left(\begin{array}{lll}
2 & 0 & 1 \\
0 & 2 & 2 \\
1 & 2 & 3
\end{array}\right)
$$

There are six possible CI statements over the ground set $N=123=\{1,2,3\}$ :

$$
\mathcal{A}_{N}=\{(12 \mid),(12 \mid 3),(13 \mid),(13 \mid 2),(23 \mid),(23 \mid 1)\} .
$$

The three marginal CI statements (12|), (13|) and (23|) have empty conditioning sets. Their corresponding almost-principal minors are off-diagonal entries of $\Sigma$. The other three are $2 \times 2$ determinants:

$$
\begin{aligned}
& \operatorname{det} \Sigma_{12}=0 \text {, } \\
& \operatorname{det} \Sigma_{13 \mid}=1 \text {, } \\
& \operatorname{det} \Sigma_{23 \mid}=2 \text {, } \\
& \operatorname{det} \Sigma_{12 \mid 3}=\operatorname{det}\left(\begin{array}{ll}
0 & 1 \\
2 & 3
\end{array}\right)=-1, \quad \operatorname{det} \Sigma_{13 \mid 2}=\operatorname{det}\left(\begin{array}{ll}
1 & 0 \\
2 & 2
\end{array}\right)=2, \quad \operatorname{det} \Sigma_{23 \mid 1}=\operatorname{det}\left(\begin{array}{ll}
2 & 0 \\
1 & 2
\end{array}\right)=4 \text {. }
\end{aligned}
$$

Collecting all $(i j \mid K)$ which evaluate to zero gives the CI structure $\llbracket \Sigma \rrbracket=\{(12 \mid)\}$.

This example shows that the CI structure $\{(12 \mid)\}$ over ground set $N=123$ is realizable by a Gaussian distribution. Determining the CI structure of a given matrix is easy. The reverse "synthesis problem" of deciding whether a given CI structure has a realization by a Gaussian distribution involves intricate algebraic relations between the principal and almost-principal minors of a generic positive-definite matrix. An algebraic proof that a CI structure $\mathcal{A}$ is non-realizable always comes with a valid inference rule $\varphi$ for Gaussians which is not satisfied by $\mathcal{A}$, such as in the next example. 
Example 2.2 Let again $N=123$ be a three-element ground set. To check whether the CI structure $\mathcal{A}=\{(12 \mid),(12 \mid 3)\}$ is realizable by a Gaussian distribution, write a generic covariance matrix

$$
\Sigma=\left(\begin{array}{lll}
p & a & b \\
a & q & c \\
b & c & r
\end{array}\right)
$$

and consider the equations imposed on $\Sigma$ by $\mathcal{A}$ :

$$
0=\operatorname{det} \Sigma_{12 \mid}=a \quad \text { and } \quad 0=\operatorname{det} \Sigma_{12 \mid 3}=\operatorname{det}\left(\begin{array}{ll}
a & b \\
c & r
\end{array}\right)=a r-b c .
$$

Together these equations imply $b c=0$ and therefore $b=0$ or $c=0$. This proves that the inference rule

$$
(12 \mid) \wedge(12 \mid 3) \Rightarrow(13 \mid) \vee(23 \mid)
$$

is valid for all Gaussian distributions. Since $\mathcal{A}$ does not satisfy this inference rule, it is not realizable by a Gaussian distribution. It is easy to see that the two inclusion-minimal CI structures which extend $\mathcal{A}$ and which are realizable by a positive-definite matrix are $\{(12 \mid),(12 \mid 3),(13 \mid),(13 \mid 2)\}$ and $\{(12 \mid),(12 \mid 3),(23 \mid),(23 \mid 1)\}$ corresponding to the two alternative conclusions of the inference rule.

A gaussoid is a subset of $\mathcal{A}_{N}$ which is closed under the gaussoid axioms:

$$
\begin{aligned}
& (i j \mid L) \wedge(i k \mid j L) \Leftrightarrow(i k \mid L) \wedge(i j \mid k L), \\
& (i j \mid k L) \wedge(i k \mid j L) \Leftrightarrow(i j \mid L) \wedge(i k \mid L), \\
& (i j \mid L) \wedge(i j \mid k L) \Rightarrow(i k \mid L) \vee(j k \mid L) \text {, }
\end{aligned}
$$

for all distinct $i, j, k \in N$ and $L \subseteq N \backslash i j k$. The first two formulas decompose into $2 \cdot 2 \cdot 2=8$ inference forms in total (some of which are redundant due to symmetries in the axioms); the third formula is a single inference form. The axiom $(\mathcal{G}$.i) defines semigraphoids, the " $\Rightarrow$ " direction of $(\mathcal{G}$.ii) is known as the intersection axiom. These six inference rules together form the definition of a graphoid. The " $\Leftarrow$ " direction of $(\mathcal{G}$.ii), the converse of intersection, is the composition axiom. The final axiom $(\mathcal{G}$.iii) is weak transitivity, a special case of which was proved in Example 2.2. Thus gaussoids are the weakly transitive, compositional graphoids, or the weakly transitive semigaussoids, as coined in [9].

The gaussoid axioms are valid inference rules for Gaussian distributions on every ground set $N$ by [22, Corollary 1]. They are the "simplest" inference rules for Gaussians in the sense that they are not only necessary but also sufficient for Gaussian realizability over the three-element ground set, which is the smallest non-trivial size. They are not sufficient for more than three variables. The main result of this paper (Corollary 3.1) shows that every valid inference rule which has at most two antecedents is implied by the gaussoid axioms - without restrictions on the finite ground set $N$.

The symmetries of Gaussian CI structures play a prominent role in the proof of the main result in Section 6. The symmetric group $S_{N}$ of permutations of $N$ acts on the random vector $\xi=\left(\xi_{i}\right)_{i \in N}$ by relabeling the entries. This immediately translates to an action on CI statements as

$$
(i j \mid K)^{\pi}:=(\pi(i j) \mid \pi(K)),
$$


for $\pi \in S_{N}$. This action is extended element-wise to CI structures. The equivalence relation induced by this group action on CI structures is isomorphy. On covariance matrices, the group acts accordingly by simultaneous permutation of the rows and columns or, equivalently, a permutation of the axes of $\mathbb{R}^{N}$. This is an orthogonal coordinate change and thus preserves positive-definiteness. Another important symmetry is the one induced by duality of CI statements,

$$
(i j \mid K)^{*}:=(i j \mid N \backslash i j K),
$$

which swaps a conditioning set $K$ with its complement in $N \backslash i j$. On covariance matrices, duality corresponds to matrix inversion $\Sigma \mapsto \Sigma^{-1}$ so that $\llbracket \Sigma^{-1} \rrbracket=\llbracket \Sigma \rrbracket^{*}$; cf. [17, Lemma 1]. Both, isomorphy and duality, are special cases of a larger symmetry group, the hyperoctahedral group $B_{N}$, which is generated by the reflection symmetries of the $N$-dimensional cube. The combinatorial motivation for considering this group is explained in [4], but it is not important for the present work. As an abstract group, $B_{N}$ equals the semidirect product $(\mathbb{Z} / 2)^{N} \rtimes S_{N}$, i.e., each of its elements can be uniquely written as a composition of a swap from $(\mathbb{Z} / 2)^{N}$ and a permutation from $S_{N}$. Each vector in the group of swaps $(\mathbb{Z} / 2)^{N}$ is an indicator vector of a subset $Z \subseteq N$ and acts on a CI statement via

$$
(i j \mid K)^{Z}:=(i j \mid K \oplus(Z \backslash i j)),
$$

where $\oplus$ denotes the symmetric difference $A \oplus B=A \backslash B \cup B \backslash A$. Duality is a special case of this action by swapping everything, i.e., $Z=N$. The second constituent of $B_{N}=$ $(\mathbb{Z} / 2)^{N} \rtimes S_{N}$ are the permutations $S_{N}$, which simply act by isomorphy.

Example 2.3 Consider the CI structure $\mathcal{A}=\{(12 \mid),(12 \mid 345),(34 \mid),(34 \mid 25)\}$. This structure satisfies the gaussoid axioms. Let $(13)$ be the cyclic permutation on $N=12345$ which exchanges 1 with 3 and leaves every other element fixed. The images of (1 3), duality and swap by $Z=123$ on $\mathcal{A}$ are, respectively:

$$
\begin{aligned}
\mathcal{A}^{(13)} & =\{(23 \mid),(23 \mid 145),(14 \mid),(14 \mid 25)\}, \\
\mathcal{A}^{*}=\mathcal{A}^{12345} & =\{(12 \mid 345),(12 \mid),(34 \mid 125),(34 \mid 1)\}, \\
\mathcal{A}^{123} & =\{(12 \mid 3),(12 \mid 45),(34 \mid 12),(34 \mid 15)\} .
\end{aligned}
$$

It is easy to see that the gaussoid axioms $(\mathcal{G} . \mathrm{i})-(\mathcal{G}$.iii) are invariant under the hyperoctahedral group. Thus, every CI structure obtained above from $\mathcal{A}$ by one of the group actions must be a gaussoid as well.

In contrast to gaussoids, realizable Gaussian CI structures are invariant only under isomorphy and duality, but not under the hyperoctahedral group. The $B_{N}$ action can be defined on symmetric matrices but it does not preserve positive-definiteness. This is explained in detail in Section 4. That section also extends the definition of realizability via $(\Perp)$ to matrices over ordered fields other than $\mathbb{R}$ and, if the field is not ordered, to matrices whose principal minors are non-zero instead of positive, giving rise to, respectively, positively and algebraically realizable gaussoids over specific fields. Algebraically realizable CI structures turn out to be invariant under the hyperoctahedral group, which is a key ingredient to the proof of our main result. 


\section{Statement of the main result}

On a fixed ground set $N$, each subset of $\mathcal{A}_{N}$ corresponds to an assignment of truth values to Boolean variables indexed by $\mathcal{A}_{N}$. Every set of CI structures (a set of truth assignments to the elements of $\mathcal{A}_{N}$ ) is the set of satisfying assignments to a Boolean formula in conjunctive normal form (CNF) whose variables are in $\mathcal{A}_{N}$. We restrict attention to the clauses of these CNFs. Each clause $\varphi$ is a disjunction of negated and non-negated statements from $\mathcal{A}_{N}$ and can be written in inference form

$$
\varphi: \bigwedge_{s}\left(i_{s} j_{s} \mid K_{s}\right) \Rightarrow \bigvee_{t}\left(x_{t} y_{t} \mid Z_{t}\right)
$$

This the general form of $\mathrm{CI}$ inference axioms, which are the subject of this article.

Definition 3.1 Let $\mathbb{A} \supseteq \mathbb{A}^{*}$ be sets of CI structures over a fixed ground set $N$. $\mathbb{A}$ is a $k$ antecedental approximation of $\mathbb{A}^{*}$ if every inference form $\varphi$ with at most $k$ antecedents and variables in $\mathcal{A}_{N}$ which is valid for $\mathbb{A}^{*}$ is also valid for $\mathbb{A}$.

We imagine $\mathbb{A}$ to be a simpler set approximating $\mathbb{A}^{*}$ from above. Because of the inclusion $\mathbb{A}^{*} \subseteq \mathbb{A}$, every inference rule which is valid for $\mathbb{A}$ also holds for $\mathbb{A}^{*}$. The definition above concerns a degree $k$ to which the converse holds. In this article, the role of $\mathbb{A}$ is played by gaussoids and that of $\mathbb{A}^{*}$ by realizable gaussoids, for different notions of realizability. From an axiomatic point of view, one may also say that the axioms for $\mathbb{A}$ are $k$-antecedentally complete for the chosen notion of realizabillity $\mathbb{A}^{*}$.

Our proof of the two-antecedental approximation property of gaussoids relies on a general principle which was also used in Studený's proof for discrete CI. A minimal $\mathbb{A}$-extension of a CI structure $\mathcal{A}$ is a CI structure $\mathcal{A}^{\prime}$ which is inclusion-minimal with the properties that $\mathcal{A}^{\prime} \supseteq \mathcal{A}$ and $\mathcal{A}^{\prime} \in \mathbb{A}$. In the world of discrete $\mathrm{CI}$ and semigraphoids, this minimal extension is unique, because both, discretely realizable CI structures and semigraphoids, are closed under intersection; but for Gaussians and gaussoids this is not true, as seen in Example 2.2.

Lemma 3.1 Let $\mathbb{A} \supseteq \mathbb{A}^{*}$ be sets of $C I$ structures over $N$. Then $\mathbb{A}$ is a $k$-antecedental approximation of $\mathbb{A}^{*}$ if every minimal $\mathbb{A}$-extension of every subset of $\mathcal{A}_{N}$ of cardinality at most $k$ belongs to $\mathbb{A}^{*}$.

Proof Let $\varphi: \bigwedge \mathcal{L} \Rightarrow \bigvee \mathcal{M}$ be a valid inference rule for $\mathbb{A}^{*}$ with $|\mathcal{L}| \leq k$. We have to show that $\varphi$ is valid for $\mathbb{A}$. Equivalently, letting $\mathbb{A}(\varphi)$ denote the subset of $\mathbb{A}$ which satisfies $\varphi$, we show that $\mathbb{A} \subseteq \mathbb{A}(\varphi)$.

Consider any $\mathcal{A} \in \mathbb{A}$. If the antecedents $\mathcal{L}$ of $\varphi$ are not contained in $\mathcal{A}$, then $\mathcal{A}$ is vacuously contained in $\mathbb{A}(\varphi)$. On the other hand, if $\mathcal{A}$ contains $\mathcal{L}$, then it also contains a minimal $\mathbb{A}$-extension $\mathcal{L}^{\prime}$ of $\mathcal{L}$. Since $|\mathcal{L}| \leq k$, the structure $\mathcal{L}^{\prime}$ belongs to $\mathbb{A}^{*}$ by assumption. Hence $\mathcal{L}^{\prime}$ satisfies $\varphi$, which means that $\mathcal{L}^{\prime} \cap \mathcal{M} \neq \emptyset$. Then $\mathcal{A}$, containing $\mathcal{L}^{\prime}$, also satisfies $\varphi$.

We can now state the main result:

Theorem 3.1 Over every ground set, every minimal gaussoid extension of at most two CI statements is realizable by a positive-definite matrix with rational entries, which can be picked arbitrarily close to the identity matrix. 
There is a notion of algebraic realizability over every field and of positive realizability over every ordered field. In each case, testing realizability of a fixed gaussoid means deciding if a system of polynomial equations, inequations and (for positivity) inequalities with integer coefficients has a solution. Since the rational numbers are the prime field of characteristic zero, finding a positive and rational solution is the hardest problem among all realizabilities over fields of characteristic zero.

The fact that the approximation is valid uniformly over all ground sets is significant. Generally, the realizabilities considered here do not have a finite axiomatization. This means that, as the ground set grows, evermore logically independent inference rules become necessary to cut out the realizable gaussoids. Theorem 3.1 implies that among these new inference rules for larger and larger ground sets, the easiest ones, up to two antecedents, are all logical consequences of the well-known gaussoid axioms. This is the titular

\section{Corollary 3.1 Gaussoids are two-antecedental approximations of algebraic and of positive Gaussian conditional independence structures over characteristic zero.}

The realizability proofs for Theorem 3.1 are composed of two ideas to be presented in the next two sections, respectively. The first idea is to relax the positive-definiteness requirement and study instead the aforementioned algebraic Gaussians over general fields. The resulting CI structures are still gaussoids and they are closed under the action of the hyperoctahedral group which allows passing to an easier orbit representative in realizability proofs. The second idea, compensating the previous relaxation, is to study more special realizations, namely rational parametrizations of spaces of matrices. Formally, such a space is represented by a matrix whose entries are elements of the rational function field $\mathbb{Q}\left(\varepsilon_{1}, \ldots, \varepsilon_{p}\right)$ with infinitesimal variables $\varepsilon_{k}$. In this space, the algebraically realized gaussoid over $\mathbb{Q}$ is determined uniquely by the rational functions for all sufficiently small values of the $\varepsilon_{k}$. If the parametrized matrices converge to a positive-definite matrix as the $\varepsilon_{k}$ tend to zero, then matrices in the interior of this space are positive-definite for small enough $\varepsilon_{k}$. In this way, positive realizability of a gaussoid is recovered from an algebraic construction and inspection of a limit. In the proof of the main theorem in Section 6, both techniques are applied to minimal gaussoid extensions of at most two CI statements. We turn the problem of finding positive-definite realizations around and instead find spaces of matrices realizing one easy hyperoctahedral representative of each gaussoid orbit converging to every hyperoctahedral image of the identity matrix. Then, given any gaussoid in the representative's orbit, we apply the inverse group action to the right space of matrices so that the transformed space approaches the positive-definite identity matrix and realizes the given gaussoid. This yields the desired rational regular Gaussian realizations.

\section{Algebraic Gaussians and the hyperoctahedral group}

The gaussoid axioms were derived in [22, Corollary 1] as consequences of an identity among minors of a complex symmetric matrix, where the proof of each instance of an axiom requires certain principal minors of the matrix not to vanish. It follows that the gaussoid axioms hold for the set $\llbracket \Gamma \rrbracket=\left\{(i j \mid K) \in \mathcal{A}_{N}\right.$ : $\left.\operatorname{det} \Gamma_{i j \mid K}=0\right\}$ of vanishing almost-principal minors of any symmetric matrix $\Gamma$, provided that all of its principal minors are non-zero. Such matrices are principally regular. In this article positive-definite and principally regular matrices are implicitly symmetric. Moreover, $\Gamma$ can have entries from any field $\mathbb{K}$ because, while Matúš's result is stated for complex matrices only, special structure of the complex 
numbers is only invoked in a continuity argument which is circumvented by the assumption of principal regularity.

Principal regularity is not only an obvious substitute for positive-definiteness over arbitrary fields. It is the technical condition which allows the formation of all Schur complements of the matrix, which correspond to conditional distributions in the positive-definite setting. The property is inherited by the inverse matrix, by principal submatrices and Schur complements and therefore is precisely what is required to salvage the theory of minors of regular Gaussians; see [4].

Definition 4.1 A gaussoid $\mathcal{G}$ is algebraically realizable (over $\mathbb{K}$ ) if there is a principally regular matrix $\Gamma$ over $\mathbb{K}$ such that $\mathcal{G}=\llbracket \Gamma \rrbracket$. If in addition $\mathbb{K}$ is an ordered field and $\Gamma$ has only positive principal minors, then $\mathcal{G}$ is positively realizable (over $\mathbb{K}$ ). By slight abuse of language we refer to algebraically realizable gaussoids as well as their realizing matrices as algebraic Gaussians and similarly for positive Gaussians.

In this terminlogy, the familiar multivariate Gaussian distributions are positive Gaussians over the real-closed field $\mathbb{R}$. Every algebraic Gaussian is, by Matúš's corollary, a gaussoid. Conversely, given any gaussoid $\mathcal{G}$, its algebraic realization space over a field $\mathbb{K}$ is the set of matrices with entries in $\mathbb{K}$ that algebraically realize $\mathcal{G}$. It is specified by the vanishing of the almost-principal minors in $\mathcal{G}$, the non-vanishing of the almost-principal minors not in $\mathcal{G}$ and the non-vanishing of all principal minors of a symmetric matrix. The space of these matrices is a constructible set. The positive realization space over an ordered field refines the nonvanishing of principal minors into positivity constraints, resulting in a semialgebraic set.

Remark 4.1 With a fixed notion of realizability, the CI implication problem asks to decide if a given inference form is valid for the class of realizable CI structures. There is much previous work about CI implication for graphical models and approximations or special cases of discrete CI; see for instance [5, 11, 26, 39] as well as [1] from the point of view of information theory, and the references therein. For Gaussians over algebraically closed fields, there is an algebraic characterization of CI implication which was observed by Matúš. His result [22, Proposition 1] is stated for the complex numbers but again the proof works over arbitrary algebraically closed fields, with the crucial ingredient being Hilbert's Nullstellensatz. The geometric summary of this characterization is that $\bigwedge \mathcal{L} \Rightarrow$ $\bigvee \mathcal{M}$ holds if and only if in the affine space of symmetric matrices $\Gamma$, the polynomial $f_{\mathcal{M}}=\prod_{(i j \mid K) \in \mathcal{M}}$ det $\Gamma_{i j \mid K}$ vanishes on the constructible set that is defined by the vanishing of the almost-principal minors indexed by $\mathcal{L}$ and the non-vanishing of the principal minors of $\Gamma$, because then on every point on this constructible set defined by $\mathcal{L}$ at least one of the minors in $\mathcal{M}$ vanishes, proving the validity of the inference. This set of matrices is obtained as a projection of a variety in a higher-dimensional affine space by a standard construction and hence the CI implication problem amounts to a radical ideal membership test. The same idea paired with the Real Nullstellensatz yields a characterization for algebraic realizability over real-closed fields, employing the real variety and hence the real radical of the analogously defined ideal. Finally, the Positivstellensatz can be used similarly to characterize the CI implication problem for positive Gaussians over a real-closed field in terms of an ideal membership query on the radical ideal of the preorder associated to the positive realization space of $\mathcal{L}$. The reader is referred to [6] and [18] for background information on geometry over algebraically and, respectively, real-closed fields. These characterizations of CI implication directly yield a procedure for realizability testing. Namely, a CI structure $\mathcal{L}$ is realizable if and only if it does not appear as the antecedent set of a non-trivial 
valid inference rule, the weakest of which, and hence the only one that needs to be tested, is $\bigwedge \mathcal{L} \Rightarrow \bigvee\left(\mathcal{A}_{N} \backslash \mathcal{L}\right)$.

Algebraic realizations have two advantages over positive realizations. The first advantage is that algebraic realizability over algebraically closed fields tends to be easier to decide in practice, as outlined in the previous remark, using Hilbert's Nullstellensatz and Gröbner bases, than positive realizability over a real-closed field, requiring a Positivstellensatz certificate for non-realizability. Over an ordered field, a positively realizable gaussoid is always algebraically realizable.

The second advantage, paramount to the proof of our main theorem, is that algebraic realizability is preserved under the hyperoctahedral group introduced in Section 2. The hyperoctahedral group action on realizing matrices is a quotient of the group $(\mathbb{Z} / 4)^{N} \rtimes S_{N}$. This group is, in turn, a discrete subgroup of the $\mathrm{SL}_{2}(\mathbb{R})^{N}$ action on the Lagrangian Grassmannian; cf. $[3,14]$. In the semidirect product, every group element can be written as the composition of an element of $S_{N}$ and one of $(\mathbb{Z} / 4)^{N}$. The permutation part is just an orthogonal coordinate change, permuting rows and columns of the matrix, corresponding to the $S_{N}$ action on CI structures (almost-principal minors) and merely permuting the set of principal minors. This action changes neither principal regularity nor positive-definiteness and therefore we focus on the $(\mathbb{Z} / 4)^{N}$ part in the remainder of this section.

Our account of the $(\mathbb{Z} / 4)^{N}$ symmetry is based on [3, Section 3] but we give more details about the action on matrices. Each $\mathbb{Z} / 4$ factor in the $N$-fold product making up the group is represented by the four $2 \times 2$ matrices

$$
\mathbb{Z} / 4=\left\{\left(\begin{array}{ll}
1 & 0 \\
0 & 1
\end{array}\right),\left(\begin{array}{cc}
0 & 1 \\
-1 & 0
\end{array}\right),\left(\begin{array}{cc}
-1 & 0 \\
0 & -1
\end{array}\right),\left(\begin{array}{cc}
0 & -1 \\
1 & 0
\end{array}\right)\right\} .
$$

To each tuple $\left(X_{1}, \ldots, X_{n}\right) \in(\mathbb{Z} / 4)^{N}$ we associate four $N \times N$ diagonal matrices $A, B, C, D$ such that

$$
X_{i}=\left(\begin{array}{cc}
A_{i i} & B_{i i} \\
C_{i i} & D_{i i}
\end{array}\right)
$$

The image of a symmetric matrix $\Gamma$ under $\left(X_{1}, \ldots, X_{n}\right)$ is $\Gamma^{\prime}=(A+\Gamma C)^{-1}(B+\Gamma D)$. $\Gamma^{\prime}$ is again symmetric by [14, Lemma 13] and the following Proposition 4.1 describes its principal and almost-principal minors. To facilitate this description we use a parametrization of this group action. For any subset $Z \subseteq N$ and a tuple of signs $\delta \in\{ \pm 1\}^{N}$, choose the group element $\left(X_{1}, \ldots, X_{n}\right)$ where

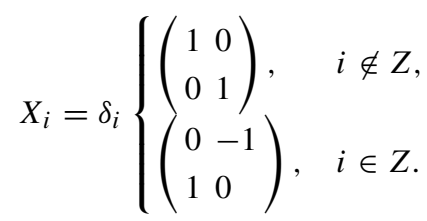

Then the action can be written as $\mathcal{S}_{Z}^{\delta}(\Gamma):=(A+\Gamma C)^{-1}(B+\Gamma D)$ with

$$
A_{i i}=D_{i i}=\left\{\begin{array}{ll}
\delta_{i}, & i \notin Z, \\
0, & i \in Z,
\end{array} \quad C_{i i}=-B_{i i}= \begin{cases}0, & i \notin Z, \\
\delta_{i}, & i \in Z .\end{cases}\right.
$$

In expressing minors of $\mathcal{S}_{Z}^{\delta}(\Gamma)$ in terms of $Z, \delta$ and $\Gamma$, it becomes necessary to recombine the involved subsets of $N$. Using the abbreviations $A B=A \cup B$ and $[A B]=A \cap B$ as well as $A^{\mathrm{C}}$ for the complement of $A$ in $N$, any combination of interest can be efficiently written 
down in "disjunctive normal form". For example, $\left[Z K^{\mathrm{c}}\right]\left[Z^{\mathrm{c}} K\right]=\left(Z \cap K^{\mathrm{c}}\right) \cup\left(K \cap Z^{\mathrm{c}}\right)=$ $(Z \backslash K) \cup(K \backslash Z)=Z \oplus K$.

One subtlety in the following statement and its proof concerns the signs of minors. In order to have a well-defined sign of the determinant, we employ the following sign convention about the ordering of rows and columns in submatrices. Because the sign is invariant under simultaneous permutations of the rows and columns, the absolute ordering is not important, but instead which row is matched with which column in any ordering of the row and column sets. Instead of imposing an absolute order on the set $N$, it will be convenient to pair every $k \in K$ with itself, in principal minors with respect to $K$, and in almost-principal minors $(i j \mid K)$ to additionally pair $i$ and $j$.

Proposition 4.1 Let $\Gamma$ be principally regular over $\mathbb{K}$ and $Z \subseteq N$ and $\delta \in\{ \pm 1\}^{N}$ be arbitrary. Then $\Gamma^{\prime}=\mathcal{S}_{Z}^{\delta}(\Gamma)$ is principally regular over $\mathbb{K}$. The gaussoid $\llbracket \Gamma^{\prime} \rrbracket=\llbracket \Gamma \rrbracket^{Z}=$ $\{(i j \mid(Z \backslash i j) \oplus K):(i j \mid K) \in \llbracket \Gamma \rrbracket\}$. More precisely, we have the following formulas for the principal and almost-principal minors of $\Gamma^{\prime}$ :

$$
\begin{aligned}
\Gamma_{K}^{\prime} & =(-1)^{[Z K]} \operatorname{det} \Gamma_{Z}^{-1} \cdot \operatorname{det} \Gamma_{Z \oplus K}, \\
\Gamma_{i j \mid K}^{\prime} & =(-1)^{[Z K]} \operatorname{det} \Gamma_{Z}^{-1} \cdot \delta_{i} \delta_{j} \operatorname{det} \Gamma_{i j \mid(Z \backslash i j) \oplus K} .
\end{aligned}
$$

Proof The matrix $\Gamma^{\prime}$ satisfies the matrix equation $(A+\Gamma C) \Gamma^{\prime}=B+\Gamma D$ and hence its minors can be computed with a generalized Cramer's rule [12]:

$$
\operatorname{det} \Gamma_{I, J}^{\prime}=\operatorname{det}(A+\Gamma C)^{-1} \operatorname{det}[(A+\Gamma C)(I, J: B+\Gamma D)],
$$

for sets $I, J \subseteq N$ of the same size and where $X(I, J: Y)$ denotes the matrix $X$ where the columns indexed by $I$ are replaced by the columns of $Y$ indexed by $J$. In this notation, we omit $J$ if it equals $I$. In addition we use $\delta X$ to denote the matrix $X$ where the $i$ th column is scaled with $\delta_{i}$.

By definition of $A$ and $C$ we have $A+\Gamma C=\delta \Gamma\left(Z^{\mathrm{C}}: I_{N}\right)$ and by Laplace expansion on the unit columns in $Z^{\mathrm{C}}$,

$$
\operatorname{det}(A+\Gamma C)=\prod_{k} \delta_{k} \cdot \operatorname{det} \Gamma_{Z}
$$

To compute the principal minor for $K \subseteq N$, notice that

$$
(A+\Gamma C)(K: B+\Gamma D)=\delta\left[\Gamma\left(Z^{\mathrm{C}}: I_{N}\right)\right]\left(K: \Gamma\left(Z:-I_{N}\right)\right)=: \delta \Gamma^{\prime \prime} .
$$

The columns of $\Gamma^{\prime \prime}$ are composed as follows:

$\left[Z K^{\mathrm{c}}\right]:$ respective columns of $\Gamma$,

$[Z K]$ : respective negative unit vectors,

$\left[Z^{\mathrm{c}} K^{\mathrm{c}}\right]$ : respective unit vectors,

$\left[Z^{\mathrm{C}} K\right]$ : respective columns of $\Gamma$.

By Laplace expansion of the unit vector columns, we obtain

$$
\operatorname{det} \delta \Gamma^{\prime \prime}=\prod_{k} \delta_{k} \cdot(-1)^{[Z K]} \operatorname{det} \Gamma_{\left[Z K^{\mathrm{c}}\right]\left[Z^{\mathrm{c}} K\right]},
$$

and $\left[Z K^{\mathrm{c}}\right]\left[Z^{\mathrm{c}} K\right]=Z \oplus K$ proves the principal minor formula. Notice that the Laplace expansions deleted the same rows and columns, so the sign convention is preserved.

For the almost-principal minor given by $(i j \mid K)$, the same procedure yields the columns of $\Gamma^{\prime \prime}$ 
$\left[Z(i K)^{\mathrm{c}}\right]: \quad$ respective columns of $\Gamma$,

$[Z K]: \quad$ respective negative unit vectors,

$\left[Z^{\mathrm{c}}(i K)^{\mathrm{c}}\right]$ : respective unit vectors,

$\left[Z^{\mathrm{c}} K\right]:$ respective columns of $\Gamma$,

$i$ : the $j$ th column of $\Gamma\left(Z:-I_{N}\right)$.

Pulling out the $\delta$ signs from the determinant, we get the sign $\prod_{k \neq i, j} \delta_{k} \cdot \delta_{j}^{2}=\delta_{i} \delta_{j} \prod_{k} \delta_{k}$ because the $i$ th column's $\delta_{i}$ was replaced by a $j$ th column's $\delta_{j}$. In addition, performing again Laplace expansion on the unit vector columns, the determinant so far is

$$
\operatorname{det} \delta \Gamma^{\prime \prime}=\delta_{i} \delta_{j} \prod_{k} \delta_{k} \cdot(-1)^{[Z K]} \operatorname{det} \Gamma_{i\left[Z(i K)^{\mathrm{c}}\right]\left[Z^{\mathrm{c}} K\right]}^{\prime \prime} \cdot
$$

The contents of column $i$ of $\Gamma^{\prime \prime}$ depends on whether $j \in Z$ or not. If $j \notin Z$, then the $i$ th column of $\Gamma^{\prime \prime}$ is just the $j$ th column of $\Gamma$. Thus the remaining minor of $\Gamma^{\prime \prime}$ in $(\diamond)$ is revealed to be the almost-principal minor of $\Gamma$ with row indices $i\left[Z(i K)^{\mathrm{c}}\right]\left[Z^{\mathrm{c}} K\right]=i\left[Z(i j K)^{\mathrm{c}}\right]\left[Z^{\mathrm{c}} K\right]$ and column indices $j\left[Z(i j K)^{\mathrm{c}}\right]\left[Z^{\mathrm{c}} K\right]$. It is easy to see that the replacement of column $i$ by column $j$ leaves the rows and columns correctly paired and it remains to compute the conditioning set as $\left[Z(i j K)^{\mathrm{C}}\right]\left[Z^{\mathrm{c}} K\right]=(Z \backslash i j) \oplus K$.

If $j \in Z$, then the $i$ th column contains the negative $j$ th unit vector. Laplace expansion with respect to this column results in the column labeled $i$ and the row labeled $j$ to be removed and incurs a sign change which depends on the distance between these columns. By simultaneously reordering rows and columns, we can assume that rows and columns $i$ and $j$ are next to each other. In this case, the sign change is -1 , which is compensated by the entry -1 in the eliminated column. The reordering ensures that rows and columns are properly paired after Laplace expansion. The remaining minor of $\Gamma^{\prime \prime}$ has row indices $i\left[Z(i K)^{\mathrm{c}}\right]\left[Z^{\mathrm{c}} K\right] \backslash j=i\left[Z(i j K)^{\mathrm{c}}\right]\left[Z^{\mathrm{c}} K\right]$ and column indices $\left[Z(i K)^{\mathrm{c}}\right]\left[Z^{\mathrm{c}} K\right]=$ $j\left[Z(i j K)^{\mathrm{c}}\right]\left[Z^{\mathrm{c}} K\right]$ and is thus again the almost-principal minor $(i j \mid(Z \backslash i j) \oplus K)$ of $\Gamma$.

These formulas describe in particular all the entries of $\mathcal{S}_{Z}^{\delta}(\Gamma)$ in terms of $\Gamma, Z$ and $\delta$. Remarkably, the choice of $\delta$ has no influence at all on the principal minors, and only changes the sign of almost-principal ones. Hence, by identifying in each $\mathbb{Z} / 4$ factor the two matrices with opposite signs, we obtain a quotient group isomorphic to $(\mathbb{Z} / 2)^{N} \rtimes S_{N}$ which faithfully implements the hyperoctahedral group on algebraic gaussoids over any field. The realizing matrix may not be well-defined but the quotient is conclusive about its positivity, over ordered fields, and thus can be used to certify positive realizability of hyperoctahedral images of gaussoids.

Remark 4.2 If $\Gamma$ is a principally regular matrix over a field $\mathbb{K}$ and the diagonal entries $\Gamma_{i i}$ are squares in $\mathbb{K}$, then the diagonal matrix $D$ with entries $1 / \sqrt{\Gamma_{i i}}$ can be formed over $\mathbb{K}$. It is easy to check that $D \Gamma D$ is a principally regular matrix which realizes the same gaussoid as $\Gamma$ and has a 1-diagonal. This argument allows to remove degrees of freedom from the generic candidate matrix in realizability tests.

For example, since the complex numbers are closed under square roots, every algebraic Gaussian over $\mathbb{C}$ has a realization with unit diagonal. The real numbers are missing $\sqrt{-1}$, so algebraic realizations of gaussoids over $\mathbb{R}$ can only be assumed to have \pm 1 entries on the diagonal. The restriction to positive realizations restores the expectation of a 1-diagonal. Finally, the rational numbers are missing many square roots, but over $\mathbb{Q}(\sqrt{-1})$ one can at least assume positive squarefree integers on the diagonal of an algebraic realization. 


\section{Infinitesimally perturbed realizations}

The theme of this section is perturbing principally regular matrices to find related but more generic CI structures in their neighborhood. By "more generic" we mean that fewer almost-principal minors vanish, which implies smaller gaussoids. Our target, the minimal gaussoid extensions of at most two CI statements, are evidently among the smallest possible gaussoids. The focus lies on perturbing the least generic matrices from this point of view (modulo the algebraic torus action described in Remark 4.2), which lie in the hyperoctahedral orbit of the identity matrix. Formally, our main tool consists of passing to Gaussians over the (ordered) field of rational functions $\mathbb{K}\left(\varepsilon_{1}, \ldots, \varepsilon_{p}\right)$, extending $\mathbb{K}$ with finitely many (infinitesimal) variables. This technique already appears in [17, Theorem 1] as well as [30, Theorem 3.3] and is on vivid display in [17, Table 1] — but always without the formalization of the concept of positive or algebraic Gaussians over function fields.

By Tarski's Transfer principle [18, Theorem 1.4.2] and the fact that Gaussian realization spaces are described by polynomial constraints with integer coefficients, every gaussoid which is algebraically or positively realizable over an ordered field extension of $\mathbb{R}$ is also algebraically or, respectively, positively realizable over $\mathbb{R}$. This principle applies to the constructions in this section, but the field of rational functions is special: if a realization over $\mathbb{Q}\left(\varepsilon_{1}, \ldots, \varepsilon_{p}\right)$ is found, then plugging in sufficiently small rational numbers for the $\varepsilon_{k}$ yields even a rational realization which is not promised by the Transfer principle. Therefore this section circumvents the Transfer principle via Lemma 5.1 and emphasizes rational constructions.

Lemma 5.1 Consider the following two situations:

(1) $\mathbb{K}$ is an infinite field and $\mathbb{L}=\mathbb{K}\left(x_{1}, \ldots, x_{p}\right)$ the field of rational functions in variables $x_{1}, \ldots, x_{p}$ over $\mathbb{K}$.

(2) $\mathbb{K}$ is an ordered field and $\mathbb{L}=\mathbb{K}\left(\varepsilon_{1}, \ldots, \varepsilon_{p}\right)$ the ordered field of rational functions in infinitesimals $0<\varepsilon_{1}<\cdots<\varepsilon_{p}$ over $\mathbb{K}$.

In both situations, a gaussoid is realizable over $\mathbb{L}$ if and only if it is already realizable over $\mathbb{K}$.

Proof One inclusion is obvious by the inclusion of fields. In the other direction, it suffices to show how to adjoin one variable $x$ or one infinitesimal $\varepsilon$, so the proof proceeds by induction on $p$.

(1) Let $\mathbb{K}$ be an infinite field and $\Gamma$ principally regular over $\mathbb{L}=\mathbb{K}(x)$. The CI structure $\llbracket \Gamma \rrbracket$ is defined by vanishing and non-vanishing constraints on principal and almostprincipal minors of $\Gamma$. These are polynomials in the entries of $\Gamma$ and therefore rational functions over $\mathbb{K}$. If a rational function $f \in \mathbb{L}$ is zero in $\mathbb{L}$, then every evaluation $f(a)$ for $a \in \mathbb{K}$ is zero. Otherwise $f$ has non-zero numerator and denominator, which are univariate polynomials over $\mathbb{K}$. The zeros of the denominator are poles of the evaluation of $f$ and the zeros of the numerator are the zeros of $f$. Both zero sets are finite. Since $\mathbb{K}$ is infinite, one can find a point $a \in \mathbb{K}$ avoiding all the undesirable poles and zeros of all principal minors and of all non-zero almost-principal minors of $\Gamma$ simultaneously. On this point $a$, the evaluation $\Gamma(a)$ is a principally regular matrix over $\mathbb{K}$ and $\llbracket \Gamma \rrbracket=\llbracket \Gamma(a) \rrbracket$.

(2) Suppose $\mathbb{K}$ is ordered. This implies that its characteristic is zero and in particular that it is infinite. Let $\Gamma$ positively realize a CI structure over $\mathbb{L}=\mathbb{K}(\varepsilon)$. Again we seek a 
positive realization of $\llbracket \Gamma \rrbracket$ over $\mathbb{K}$ by plugging in elements of $\mathbb{K}$ for $\varepsilon$. By the previous part of the proof, the "algebraic part" of positive realizability, i.e., the vanishing and non-vanishing conditions of almost-principal minors, is satisfied on all but finitely many points. It remains to find infinitely many points of $\mathbb{K}$ on which all principal minors of $\Gamma$ evaluate to positive elements of $\mathbb{K}$. By the hypothesis, the principal minors of $\Gamma$ are positive in the ordering of $\mathbb{L}$. We can assume that numerators and denominators are both positive. By the ordering of $\mathbb{L}$, a polynomial $f \in \mathbb{K}[\varepsilon]$ is positive if and only if its lowest-degree non-zero coefficient is positive in $\mathbb{K}$. It is then an easy exercise in ordered fields to construct $a_{f}>0$ in $\mathbb{K}$, depending on the coefficients and degree of $f$, such that every evaluation of $f$ on the open interval $\left(0, a_{f}\right)$ is positive in $\mathbb{K}$. Let $a^{*}$ be the minimum of the finitely many $a_{f}$ constructed for all the (numerators and denominators of ) principal minors of $\Gamma$. Since $a^{*}>0$, the interval $\left(0, a^{*}\right)$ is infinite, and all but finitely many evaluations of $\Gamma$ on this interval yield a positive realization of $\llbracket \Gamma \rrbracket$ over $\mathbb{K}$.

Remark 5.1 The proof of the first part works, moreover, for all finite fields of sufficient size. A lower bound can be given based on the number of roots that the univariate polynomials in question have, which can be bounded by the size of a concrete realizing matrix and the maximal degree of its entries.

For geometric intuition, suppose that $\mathbb{K}=\mathbb{R}$ for the moment. Inspection of the previous proof then paints the following picture of our main construction technique: we define a space of real matrices parametrized by rational functions in variables $\varepsilon_{1}, \ldots, \varepsilon_{p}$. In fact, we can replace all infinitesimals by sufficiently large and far apart powers of a single infinitesimal and imagine just a curve segment of matrices parametrized by $\varepsilon$. By the defining rational functions, we control the algebraically realized CI structure on this curve, and as $\varepsilon$ tends to zero, the matrices may approach a limit matrix whose principal regularity or positivedefiniteness carries over to them by continuity. In this way a certificate for algebraic or positive realizability of the $\mathrm{CI}$ structure on the curve over the base field $\mathbb{K}$ is obtained.

The appeal to continuity and limits can be avoided by an easy sufficient condition which is the subject of the next definition and which holds for all our later applications. Let $\mathbb{L}$ be a rational function field over $\mathbb{K}$ as in Lemma 5.1 and $\Gamma$ a matrix over $\mathbb{L}$. Suppose that the denominators of all of its entries have a non-zero constant term. Then the evaluation $\Gamma^{\circ}:=\Gamma(0, \ldots, 0)$ is a matrix over $\mathbb{K}$. Notice that each minor of $\Gamma^{\circ}$ is the constant term of the corresponding minor of $\Gamma$ and thus principal regularity and positive-definiteness of $\Gamma^{\circ}$ over $\mathbb{K}$ imply that of $\Gamma$ over $\mathbb{L}$, allowing application of Lemma 5.1.

Definition 5.1 Let $\mathbb{K}$ be a field and $\Gamma_{0}$ a principally regular matrix. A gaussoid $\mathcal{G}$ is realizable near $\Gamma_{0}$ if there exists $\Gamma$ over $\mathbb{K}\left(\varepsilon_{1}, \ldots, \varepsilon_{p}\right)$ such that $\mathcal{G}=\llbracket \Gamma \rrbracket$ and $\Gamma^{\circ}=\Gamma_{0}$. If $\Gamma$ can be chosen over the prime field $\mathbb{K}=\mathbb{Q}$, we add the adverb rationally for additional emphasis.

Among all (ordered) fields of characteristic zero, rational (positive) realizability is the most difficult to achieve, so whenever this is possible it is highlighted in the results below. Realizability near a positive-definite matrix immediately implies positive realizability by Lemma 5.1. We now assume that $\mathbb{K}$ is infinite or ordered and apply this lemma to prove general construction methods for Gaussians which reduce the case work in Section 6. 
Lemma 5.2 Let $\mathcal{G}=\llbracket \Sigma \rrbracket$ and $\mathcal{H}=\llbracket \Gamma \rrbracket$ be algebraic Gaussians over infinite $\mathbb{K}$ on disjoint ground sets $N$ and $M$, respectively. Then $\mathcal{G} \cup \mathcal{H}$ is an algebraic Gaussian over $\mathbb{K}$ on the ground set $N \cup M$. It is realizable near the block-diagonal matrix containing $\Sigma$ and $\Gamma$ blocks.

Proof Define an $(N M \times N M)$-matrix

$$
\Phi=\left(\begin{array}{cc}
\Sigma & \varepsilon \\
\varepsilon^{T} & \Gamma
\end{array}\right)
$$

where $\Sigma$ sits in the $N \times N$ block, $\Gamma$ in the $M \times M$ block and $\varepsilon=\left(\varepsilon_{i j}\right)_{i j \in N \times M}$ consists of independent variables. Obviously $\Phi^{\circ}$ is the block-diagonal matrix from the statement of the lemma and $\Phi$ has coefficients in $\mathbb{K}$. The matrix $\Phi$ defines a realizable gaussoid $\llbracket \Phi \rrbracket$ which restricts to $\mathcal{G}$ on $N$ and to $\mathcal{H}$ on $M$. It remains to see that $\llbracket \Phi \rrbracket$ contains no other CI statement $(i j \mid K)$ where we decompose $K=N^{\prime} M^{\prime}$ with $N^{\prime} \subseteq N, M^{\prime} \subseteq M$. We apply Lemma 5.1 and show that $\operatorname{det} \Phi_{i j \mid K}$ vanishes in $\mathbb{K}\left(\varepsilon_{i j}\right)$ only if $\mathcal{G}$ or $\mathcal{H}$ mandate it.

First assume $i j \subseteq N$ and $M^{\prime} \neq \emptyset$ (as $M^{\prime}=\emptyset$ yields that $\operatorname{det} \Phi_{i j \mid K}=\operatorname{det} \Sigma_{i j \mid N^{\prime}}$, whose vanishing depends only on $\mathcal{G}$ ). The almost-principal submatrix $\Phi_{i j \mid K}$ is written with rows labeled in order by $i N^{\prime} M^{\prime}$ and columns $j N^{\prime} M^{\prime}$ :

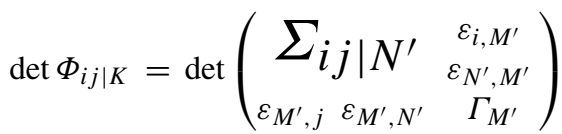

$$
\begin{aligned}
& =\operatorname{det}\left(\Gamma_{M^{\prime}}\right) \operatorname{det}\left(\Sigma_{i j \mid N^{\prime}}-\left(\begin{array}{c}
\varepsilon_{i, M^{\prime}} \\
\varepsilon_{N^{\prime}, M^{\prime}}
\end{array}\right) \Gamma_{M^{\prime}}^{-1}\left(\varepsilon_{M^{\prime}, j} \varepsilon_{M^{\prime}, N^{\prime}}\right)\right) .
\end{aligned}
$$

The first determinant is a principal minor of $\Gamma$ and hence non-zero. It suffices to show that the determinant of the Schur complement expression is not the zero polynomial. For row $a \in i N^{\prime}$ and column $b \in j N^{\prime}$ the corresponding entry of the Schur complement is

$$
\Sigma_{a b}-\sum_{k, l \in M^{\prime}}\left(\Gamma_{M^{\prime}}^{-1}\right)_{k l} \varepsilon_{a l} \varepsilon_{b k}
$$

and hence by the Leibniz formula the determinant equals

$$
\sum_{\substack{\sigma: i N^{\prime} \rightarrow j N^{\prime} \\ \text { bijective }}} \operatorname{sgn}(\sigma) \prod_{a \in i N^{\prime}}\left(\Sigma_{a \sigma(a)}-\sum_{k, l \in M^{\prime}}\left(\Gamma_{M^{\prime}}^{-1}\right)_{k l} \varepsilon_{a l} \varepsilon_{\sigma(a) k}\right) .
$$

By assumption there exists $m \in M^{\prime}$. In this multivariate polynomial the coefficient of $\varepsilon_{i m} \varepsilon_{j m}$ is

$$
\pm \sum_{\sigma \in S_{N^{\prime}}} \operatorname{sgn}(\sigma)\left(\Gamma_{M^{\prime}}^{-1}\right)_{m m} \prod_{a \in N^{\prime}} \Sigma_{a \sigma(a)}= \pm\left(\Gamma_{M^{\prime}}^{-1}\right)_{m m} \operatorname{det} \Sigma_{N^{\prime}} \neq 0
$$

which shows that $(i j \mid K) \notin \llbracket \Phi \rrbracket$ by Lemma 5.1 in case $i j \subseteq N$ and $M^{\prime} \neq \emptyset$. The same proof applies to the symmetric case with $N$ and $M$ exchanged.

The remaining case has $i \in N$ and $j \in M$. Then, by Laplace expansion

$$
\operatorname{det} \Phi_{i j \mid K}=\operatorname{det}\left(\begin{array}{cc}
\varepsilon_{i j} & \cdots \\
\vdots & \Phi_{K}
\end{array}\right)=\varepsilon_{i j} \operatorname{det} \Phi_{K} \mp \ldots,
$$

where $\operatorname{det} \Phi_{K}$ is non-zero and all other terms do not involve the variable $\varepsilon_{i j}$. 
Lemma 5.3 Let $\mathbb{K}$ be infinite and $\mathcal{G}=\llbracket \Gamma \rrbracket$ an algebraic Gaussian over $\mathbb{K}$ on $N$. If $L \cap$ $N=\emptyset$, then $\left\{(i j \mid K) \in \mathcal{A}_{N L}:(i j \mid K) \in \mathcal{G}\right\}$ and $\left\{(i j \mid K L) \in \mathcal{A}_{N L}:(i j \mid K) \in \mathcal{G}\right\}$ are both algebraic Gaussians over $\mathbb{K}$ on $N L$. They are realizable near every block-diagonal matrix whose $N$-block is $\Gamma$ and whose L-block is principally regular.

Proof The first assertion follows directly from Lemma 5.2 using that the empty gaussoid on $L$ is realizable near every principally regular matrix, by introducing independent variables for each entry. To show the second assertion, we make use of duality: if $\mathcal{G}=\llbracket \Gamma \rrbracket$ for $\Gamma$ principally regular, then $\Gamma^{-1}$ is principally regular and realizes the dual gaussoid $\llbracket \Gamma^{-1} \rrbracket=$ $\mathcal{G}^{*}=\{(i j \mid N \backslash i j K):(i j \mid K) \in \mathcal{G}\}$; cf. [3,17]. Thus we may take $\mathcal{G}^{*}$ over $N$ and embed it into $N L$ by the first part of the proof, denoting the result by $\overline{\mathcal{G}^{*}}$, and take its dual over $N L$. All these operations preserve algebraic realizability near a chosen block-diagonal matrix and we get

$$
(i j \mid K) \in \mathcal{G} \Leftrightarrow(i j \mid N \backslash i j K) \in \mathcal{G}^{*} \Leftrightarrow(i j \mid N L \backslash i j K L) \in \overline{\mathcal{G}^{*}} \Leftrightarrow(i j \mid K L) \in \overline{\mathcal{G}}^{*} .
$$

No other CI statements over $N L$ arise because duality and embedding preserve also cardinality.

Remark 5.2 The "dependent sum" construction from Lemma 5.2 can be seen as a perturbation of the direct sum of semigraphoids $[19,21]$ which is recovered in the limit $\varepsilon_{i j}=0$. Both constructions preserve the properties of being a gaussoid and being a realizable gaussoid for all the various notions of "realizable" covered in the above statements. The dependent sum yields the most generic gaussoid on $N M$ which restricts to its summands on $N$ and $M$, in that it satisfies no additional relations. For realizable gaussoids, this corresponds to the "most dependent" joint Gaussian. Similarly, Lemma 5.3 shows that marginalization and conditioning of realizable gaussoids from $N L$ to $N$ can be inverted generically, inducing no further independencies over $N L$.

It easily follows from Proposition 4.1 that the quotient action $\mathcal{S}_{Z}$ on the $(\mathbb{Z} / 4)^{N}$-orbit of the identity matrix, where components $X_{i}$ with different signs $\delta_{i}$ are identified, produces well-defined matrices, independent of the choice $\delta_{i}$ of representatives. This orbit consists of all $2^{n}$ diagonal matrices with entries $( \pm 1, \ldots, \pm 1)$. For any matrix $J$ in this orbit the action $\mathcal{S}_{Z}(J)$ flips the signs of the diagonal entries of $J$ indicated by $Z$. The action of $S_{N}$ does not leave this set of matrices either, so it constitutes an orbit under the hyperoctahedral group $B_{N}$.

Realizability near the identity matrix or its hyperoctahedral images is a well-behaved notion in the theory of CI structures: take a principally regular matrix $\Gamma$ over $\mathbb{K}\left(\varepsilon_{1}, \ldots, \varepsilon_{p}\right)$ with $\Gamma^{\circ}$ in this orbit. By Proposition 4.1, the hyperoctahedral action produces a principally regular matrix $\Delta$ over the same field such that $\Delta^{\circ}$ belongs to the hyperoctahedral orbit of the identity as well. The dependent sum in Lemma 5.2, duality, marginalization and conditioning and their reversals in Lemma 5.3 of algebraic Gaussians preserve realizability near a hyperoctahedral image of the identity over their respective ground sets. These facts and the following proposition are the main realizability tools for the most complicated cases in the next section.

Proposition 5.1 If a gaussoid $\mathcal{G}$ is (rationally) realizable near every one of the $2^{n}$ hyperoctahedral images of the identity matrix, then every hyperoctahedral image of $\mathcal{G}$ is (rationally) near-identity realizable, in particular positively realizable. 
Proof Let $\mathcal{H}$ be in $\mathcal{G}$ 's hyperoctahedral orbit, arising from $\mathcal{G}$ by a swap and a permutation. $\mathcal{H}$ is realizable near the identity if and only if $\mathcal{G}$ is realizable near the matrix which is obtained from the identity by permuting and swapping in reverse. These operations result in a hyperoctahedral image of the identity near which $\mathcal{G}$ is realizable by assumption. The hyperoctahedral action which transports this curve of realizations back to realize $\mathcal{H}$ near the identity does not change the field, so rationality is preserved.

\section{Proof of the main theorem}

In this section a proof of our main result Theorem 3.1 is delivered in the form of a series of lemmas which each settle one type of minimal gaussoid extension of at most two CI statements. In each case we construct a rational near-identity realization.

\section{Lemma 6.1 All CI structures with at most one element are rationally realizable near the} identity.

Proof The empty structure is realized by a symmetric matrix with 1-diagonal and independent variables in the off-diagonal entries. Clearly, none of the almost-principal minors of this matrix vanish as polynomials. Every singleton subset of $\mathcal{A}_{N}$ is vacuously a gaussoid. The singleton gaussoids form a single orbit under the action of the hyperoctahedral group. While this action does not in general preserve positive realizability, we can emulate it using Lemma 5.3 in a way that shows that it $i$ preserved in this case. Given any singleton $(i j \mid K)$, first permute it to $\left(12 \mid K^{\prime}\right)$, marginalize to $12 K^{\prime}$ and then contract $K^{\prime}$ to arrive at the singleton (12|) over the ground set $N=12$. These transformations preserve rational positive realizability and their inverses do as well. Thus we can transform every singleton into every other singleton while preserving realizability and it remains to see that $\{(12 \mid)\}$ is rationally positively realizable, which is trivial.

From now on we consider two-element sets $\{(i j \mid N),(k l \mid M)\}$ and their minimal gaussoid extensions. Using the fact that marginalizations and conditionings of Gaussians are Gaussians (over the same field) and that we can undo these operations generically via Lemma 5.3 , we can assume that we work over the ground set $i j k l N M$ and that $N \cap M=\emptyset$.

The gaussoid axioms have two antecedents. Every antecedent set of a gaussoid axiom is therefore not a gaussoid. The following lemma deals with this type:

Lemma 6.2 If $\mathcal{B}=\{(i j \mid N),(k l \mid M)\}$ is not a gaussoid, then each of its minimal gaussoid extensions has cardinality four and is rationally realizable near the identity.

Proof $\mathcal{B}$ not being a gaussoid requires that $(i j \mid N)$ and $(k l \mid M)$ are distinct and form the antecedent set of a gaussoid axiom. Thus the two CI statements lie in a 3-face of the ambient $i j k l N M$-cube; see [4]. We can therefore reduce the study of gaussoid extensions of $\mathcal{B}$ to this 3 -face and hence, after conditioning, to a 3-element ground set. Every gaussoid closure of $\mathcal{B}$ is thus a 3-gaussoid which is placed in a 3-face of the ijklNM-cube. With two generators, each closure has exactly four elements. The 3-gaussoids are all realizable as undirected graphical models or their duals. Rational near-identity realizations have been constructed in [17, Theorem 1] and those are embedded back into the i jklN M-cube via Lemma 5.3.

The remaining type of gaussoids is comprised of pairs of so-called inferenceless generators with respect to the gaussoid axioms: two-element subsets of $\mathcal{A}_{N}$ which are vacuously 
gaussoids. We expect this type to be the hardest to realize. The gaussoid axioms, as the previous proof shows, govern inferences of two CI statements in a common 3-face of the hypercube. The realizabilities of inferenceless pairs prove that there are no valid twoantecedental inference rules for Gaussian CI whose antecedents lie further apart in the hypercube than in a common 3-face.

We continue to assume that the ground set is $i j k l N M$ and that $N \cap M=\emptyset$. In addition, the assumption of inferenceless generators can be expressed as

$$
|N \oplus M| \geq|i j \cap k l| .
$$

This type splits into a number of cases depending on how "entangled" $i j, k l, N$ and $M$ are, as these entanglements influence the form of a potential realizing matrix. Up to the group $\mathbb{Z} / 2 \times S_{N}$ of duality and isomorphy, which preserves rational positive realizability, and symmetries in the roles of $i j$ and $k l$, there are seven cases:

\begin{tabular}{llllllll}
\hline & 1 & 2 & 3 & 4 & 5 & 6 & 7 \\
\hline$i j \cap k l$ & $\emptyset$ & $\emptyset$ & $\emptyset$ & $\emptyset$ & $i$ & $i$ & $i j$ \\
$i j \cap M$ & $\emptyset$ & $i$ & $i$ & $i j$ & $\emptyset$ & $j$ & $\emptyset$ \\
$k l \cap N$ & $\emptyset$ & $\emptyset$ & $k$ & $\emptyset$ & $\emptyset$ & $\emptyset$ & $\emptyset$ \\
\hline
\end{tabular}

The hyperoctahedral group has a considerably wider reach. Every gaussoid $\{(i j \mid N),(k l \mid M)\}$ can be transformed into $\left\{(i j \mid),\left(k l \mid M^{\prime}\right)\right\}$, where $i j \cap M^{\prime}=\emptyset$, by swapping out $N \cup(M \cap i j)$. This action reduces the seven cases in the table above to only three cases:

- $\quad\{(i j \mid),(k l \mid M)\}$ on $i j k l M$ with $i j \cap k l M=\emptyset$,

- $\quad\{(i j \mid),(i k \mid M)\}$ on $i j k M$ with $i j \cap k M=\emptyset$,

- $\quad\{(i j \mid),(i j \mid M)\}$ on $i j M$ with $i j \cap M=\emptyset$.

Not only does $B_{N}$ decrease the number of cases, it also allows to pick simpler representatives of each case. The three representatives displayed above all contain the statement $(i j \mid)$, which only mandates that a specific entry of the realizing matrix be zero. This reduction comes at the cost of not preserving positive realizability. Using Proposition 5.1, to obtain rational positive realizability of the entire orbit, we realize the above three case representatives rationally near all the matrices which are equivalent to the identity under the hyperoctahedral action.

The first case is the union of gaussoids $\{(i j \mid)\}$ and $\{(k l \mid M)\}$ over disjoint ground sets $i j$ and $k l M$ and is already settled as an instance of Lemma 5.2 with the rational near-identity realizations constructed for singleton gaussoids in Lemma 6.1. The remaining two cases are settled by Lemmas 6.3 and 6.4 below.

Lemma 6.3 The gaussoid $\{(i j \mid),(i k \mid M)\}$ on the ground set $i j k M$ is rationally realizable near all hyperoctahedral images of the identity.

Proof We introduce the following notation:

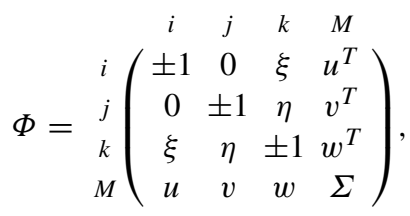


where $(i j \mid)$ is already fulfilled by imposing the zero entry. The statement $(i k \mid M)$ is equivalent to the following relation, after a Schur complement:

$$
\xi=u^{T} \Sigma^{-1} w \text {. }
$$

Thus we impose this relation on $\xi$. All other appearing symbols are supposed to be generic, i.e., $\eta$ is a variable, the vectors have independent variable entries $u_{m}, v_{m}, w_{m}$, for $m \in M$, and $\Sigma$ is a generic symmetric matrix with \pm 1 -diagonal and independent $\varepsilon_{m n}$ off-diagonals. The signs of the diagonal elements of $\Phi$ are arbitrary but fixed. $\Phi$ is a matrix over $\mathbb{Q}\left(\eta, u_{m}, v_{m}, w_{m}, \varepsilon_{m n}\right)$ whose off-diagonal entries tend to zero with the infinitesimal variables and thus it approaches any hyperoctahedral image of the identity matrix. The only denominator appears in $\xi$ and is the principal minor det $\Sigma$ with constant term \pm 1 , which is infinitesimally non-zero.

By construction, $(i j \mid)$ and $(i k \mid M)$ hold for $\Phi$. It is clear that the only interesting almostprincipal minors are those involving $\xi$. For any $N \subsetneq M$, the statement $(i k \mid N)$ surely does not hold because it is equivalent to

$$
u^{T} \Sigma^{-1} w=u_{N}^{T} \Sigma_{N}^{-1} w_{N},
$$

where the variables in $u, w, \Sigma$ are all independent. There are four remaining cases: $(i k \mid j N)$, $(i l \mid k N),(k l \mid i N)$ and $(l m \mid i k N)$, for relevant choices of $l, m$ and $N \subseteq M$.

The almost-principal minor $(i k \mid j N)$ is rewritten using Schur complement with respect to $j N$ to

$$
\operatorname{det} \Phi_{i k \mid j N}=\operatorname{det} \Phi_{j N}\left(\xi-\left(\begin{array}{ll}
0 & u_{N}^{T}
\end{array}\right) \Phi_{j N}^{-1}\left(\begin{array}{c}
\eta \\
w_{N}^{T}
\end{array}\right)\right),
$$

which vanishes if and only if the parenthesized factor vanishes as a rational function. Numerator and denominator of $\xi$ do not involve the variable $\eta$, so it suffices to show that there is a monomial divisible by $\eta$ with non-zero coefficient in the "bilinear term" inside the parentheses. All terms involving $\eta$ are

$$
\eta \sum_{n \in N} u_{n}\left(\Phi_{j N}^{-1}\right)_{j n} .
$$

Each of these summands has a unique variable $u_{n}$ which does not appear in $\Phi_{j N}$. If $N \neq \emptyset$, this ensures that the $\eta$ terms do not cancel and that the almost-principal minor does not vanish. In case $N=\emptyset$, it is sufficient to remark that $\Phi_{i k \mid}=\xi \neq 0$ because $M \neq \emptyset$ due to the assumption of inferenceless generators $(\dagger)$.

For the case $(i l \mid k N)$ first assume that $l \neq j$. Laplace expansion on the first row of the almost principal minor gives a sum

$$
\operatorname{det}\left(\begin{array}{ccc}
u_{l} & \xi & u_{N}^{T} \\
w_{l} & \pm 1 & w_{N} \\
\Sigma_{N, l} & w_{N}^{T} & \Sigma_{N}
\end{array}\right)=u_{l} \operatorname{det} \Phi_{k N} \mp \ldots
$$

of which the omitted terms are not divisible by $u_{l}$. Since the constant term of $\operatorname{det} \Phi_{k N}$ is \pm 1 , the monomial $u_{l}$ arises in the sum and cannot be canceled by other terms. If $l=j$, then $(i j \mid k N)$ is equivalent to

Again we investigate the terms divisible by $\eta$ :

$$
0=\left(\begin{array}{ll}
\xi & u_{N}^{T}
\end{array}\right) \Phi_{k N}^{-1}\left(\begin{array}{c}
\eta \\
v_{N}^{T}
\end{array}\right)
$$

$$
\eta\left(\xi\left(\Phi_{k N}^{-1}\right)_{k k}+\sum_{n \in N} u_{n}\left(\Phi_{k N}^{-1}\right)_{k n}\right)
$$


Since $\xi \neq 0$ and $\left(\Phi_{k N}^{-1}\right)_{k k}$ has constant term \pm 1 , we find the monomial $\eta u_{m} w_{m}$ for some $m \in M$ in the numerator of this almost-principal minor.

The case $(k l \mid i N)$ for $l \neq j$ is completely analogous to the previous $(i l \mid k N)$ one. In fact, the involved matrices are identical up to exchanging the places of the generic vectors $u$ and $w$, which already play symmetric roles in the definition of $\xi$. The matrix for $(k j \mid i N)$ is

$$
\left(\begin{array}{ccc}
\eta & \xi & w_{N}^{T} \\
0 & \pm 1 & u_{N}^{T} \\
v_{N} & u_{N} & \Sigma_{N}
\end{array}\right)
$$

and again Laplace expansion can be used to see that $\eta$ survives as a monomial of degree one.

The last case is $(\operatorname{lm} \mid i k N)$. If $j \notin l m$, the almost-principal minor of

$$
\left(\begin{array}{cccc}
\varepsilon_{l m} & u_{l} & w_{l} & \Sigma_{l, N} \\
u_{m} & \pm 1 & \xi & u_{N}^{T} \\
w_{m} & \xi & \pm 1 & w_{N}^{T} \\
\Sigma_{N, m} & u_{N} & w_{N} & \Sigma_{N}
\end{array}\right)
$$

has a monomial $\varepsilon_{l m}$ via Laplace expansion in the first row. The numerators of other summands in this expansion are not divisible by $\varepsilon_{l m}$, making it impossible to cancel this degree-1 monomial. Otherwise, without loss of generality, $m=j$ and the matrix

$$
\left(\begin{array}{cccc}
v_{l} & u_{l} & w_{l} & \Sigma_{l, N} \\
0 & \pm 1 & \xi & u_{N}^{T} \\
\eta & \xi & \pm 1 & w_{N}^{T} \\
v_{N} & u_{N} & w_{N} & \Sigma_{N}
\end{array}\right)
$$

is susceptible to the same Laplace expansion proof yielding a monomial $v_{l}$.

Lemma 6.4 The gaussoid $\{(i j \mid),(i j \mid M)\}$ on the ground set $i j M$ is rationally realizable near all hyperoctahedral images of the identity.

Proof We use the matrix pattern

$$
\Phi={ }_{M}^{i}\left(\begin{array}{ccc}
i & j & M \\
\pm 1 & 0 & u^{T} \\
0 & \pm 1 & v^{T} \\
u & v & \Sigma
\end{array}\right)
$$

with column vectors $u$ and $v$ and a generic matrix $\Sigma$ with \pm 1-diagonal and independent $\varepsilon_{m n}$ off-diagonals. Again, $(i j \mid)$ is imposed already by a zero entry. Unlike the situation of Lemma 6.3, we cannot make $(i j \mid M)$ hold by imposing a relation on the $i j$-entry of $\Phi$ which is already set to zero. The equation for $(i j \mid M)$ is equivalent to

$$
0=u^{T} \operatorname{adj}(\Sigma) v,
$$

that is, $u$ and $v$ are orthogonal with respect to the (infinitesimally principally regular) adjoint of $\Sigma$. Equivalently we could have used $\Sigma^{-1}$ but prefer not to introduce denominators into $\Phi$ needlessly. To enforce this relation, we define $u$ and $v$ via the Gram-Schmidt process on vectors $x$ and $y$ of mutually independent variables indexed by $M$, as follows:

$$
\begin{aligned}
u_{k} & =x_{k}, \\
v_{k} & =\alpha_{M} y_{k}-\beta_{M} x_{k},
\end{aligned}
$$

with the bilinear forms $\alpha_{L}=x_{L}^{T} \operatorname{adj}\left(\Sigma_{L}\right) x_{L}$ and $\beta_{L}=x_{L}^{T} \operatorname{adj}\left(\Sigma_{L}\right) y_{L}$ for any $L \subseteq M$. This completes the definition of $\Phi$, which is a matrix over $\mathbb{Q}\left(x_{m}, y_{m}, \varepsilon_{m n}\right)$ whose off-diagonal 
entries tend to zero with the infinitesimal variables, and clearly $\llbracket \Phi \rrbracket$ contains $(i j \mid)$ and $(i j \mid M)$. Evidently $(k l \mid N) \notin \llbracket \Phi \rrbracket$ whenever $j \notin k l N$ because the almost-principal submatrix is generic in this case. The remainder of the proof treats CI statements of the forms $(i j \mid N)$, $(j k \mid N)$ and $(k l \mid j N)$ each for all suitable $k, l$ and $N \subseteq M$.

If $N$ is any non-empty subset of $M$, the almost-principal minor $(i j \mid N)$ becomes

$$
\begin{aligned}
\operatorname{det} \Phi_{i j \mid N} & =u_{N}^{T} \operatorname{adj}\left(\Sigma_{N}\right) v_{N} \\
& =\alpha_{M} x_{N}^{T} \operatorname{adj}\left(\Sigma_{N}\right) y_{N}-\beta_{M} x_{N}^{T} \operatorname{adj}\left(\Sigma_{N}\right) x_{N} \\
& =\alpha_{M} \beta_{N}-\alpha_{N} \beta_{M} .
\end{aligned}
$$

If $N \neq M$, it suffices to find a monomial in $\alpha_{M} \beta_{N}$ which does not appear in $\alpha_{N} \beta_{M}$. Given $k \in N$ and $m \in M \backslash N$, and using that $x_{m}^{2}$ only appears in $\alpha_{M}$ via the constant term \pm 1 in the cofactor $(\operatorname{adj} \Sigma)_{m m}$, such a monomial is $x_{m}^{2} x_{k} y_{k}$.

Next consider type $(j k \mid N)$ with

$$
\operatorname{det} \Phi_{j k \mid N}=\operatorname{det} \Phi_{N}\left(\alpha_{M} y_{k}-\beta_{M} x_{k}\right)-\Phi_{j, N} \operatorname{adj}\left(\Phi_{N}\right) \Phi_{N, k} .
$$

By the assumption of inferenceless generators ( $\dagger),|M| \geq 2$, so there exists $m \in M \backslash k$. The expansion of $\alpha_{M} y_{k}$ produces the monomial $x_{m}^{2} y_{k}$ which does not appear in $\beta_{M} x_{k}$. Thus this monomial arises from the product term. The remaining term is a bilinear form with respect to $\operatorname{adj}\left(\Phi_{N}\right)$. Expanding the $\Phi_{j, N}$ vector, pretending that $x_{i}=y_{i}=0$ in case $N \ni i$, we find

$$
\Phi_{j, N} \operatorname{adj}\left(\Phi_{N}\right) \Phi_{N, k}=\alpha_{M} y_{N}^{T} \operatorname{adj}\left(\Phi_{N}\right) \Phi_{k, N}-\beta_{M} x_{N}^{T} \operatorname{adj}\left(\Phi_{N}\right) \Phi_{k, N} .
$$

Each monomial in $\alpha_{M}$ or $\beta_{M}$ has total degree at least $2 ; y_{n}, x_{n}$ and $\Phi_{k n}$ are variables or zero if $n=i \in N$. Under no circumstance does any monomial of total degree 3 arise. This proves that $x_{m}^{2} y_{k}$ is a monomial with non-zero coefficient in the expansion of $\operatorname{det} \Phi_{j k \mid N}$, hence $(j k \mid N) \notin \llbracket \Phi \rrbracket$.

The last type $(k l \mid j N)$ splits into two cases, depending on whether $i \in k l$ or not. The proofs are similar, so suppose first that $i \notin k l$. Then

$$
\operatorname{det} \Phi_{k l \mid j N}=\operatorname{det} \Phi_{j N} \varepsilon_{k l}-\Phi_{k, j N} \operatorname{adj}\left(\Phi_{j N}\right) \Phi_{j N, l} .
$$

Because det $\Phi_{j N}$ has constant term \pm 1 , the monomial $\varepsilon_{k l}$ appears in the above expansion of the almost-principal minor. It suffices to show that the bilinear form term does not produce this monomial. Indeed,

$$
\Phi_{k, j N} \operatorname{adj}\left(\Phi_{j N}\right) \Phi_{j N, l}=\sum_{a, b \in j N} \Phi_{a k} \Phi_{b l}\left(\operatorname{adj} \Phi_{j N}\right)_{a b}
$$

sums over products of three polynomials of which at most the entry of the adjoint may have a non-zero constant term. Analogously to above, this sum has no monomial of total degree 1 , so the $\varepsilon_{k l}$ monomial cannot be canceled. Finally, if $l=i$, the $\varepsilon_{k l}$ term in the calculation above becomes $x_{k}$ instead and one of the coordinates in the bilinear form term is the 0 in $\Phi_{i j \mid}$. However, this does not interfere with the argument.

\section{Remarks and examples}

We have shown that on every ground set $N$, a Boolean formula in inference form $\varphi: \bigwedge \mathcal{L} \Rightarrow$ $\bigvee \mathcal{M}$ in variables $\mathcal{A}_{N}$ and with $|\mathcal{L}| \leq 2$ is valid for all regular Gaussian distributions if and only if the gaussoid axioms on $N$ logically imply $\varphi$. The main work of the proof consists of realizing all gaussoids with two elements, as their realizability implies that no inference forms with two antecedents, beyond the deductive closure of the gaussoid axioms, are valid 
for all regular Gaussians. Since we constructed positive-definite rational realizations (in every neighborhood of the identity matrix), which is the most restrictive constellation among algebraic and positive realizations over characteristic zero, we obtain Corollary 3.1 which in character reaches slightly beyond the probabilistic origin of gaussoids and into the field of synthetic geometry.

Corollary 3.1 does not hold in general in positive characteristic. For example, it is easy to see that the only principally regular matrix over GF(2) is the identity matrix, so the GF(2)algebraic Gaussians satisfy many inference rules which are not implied by the gaussoid axioms. The proof strategy of this paper begins to fail over finite fields in Section 5. For example, Lemma 5.2 does not hold over GF(2).

The result does not generalize to more antecedents either: a valid three-antecedental inference rule for Gaussians which is not implied by the gaussoid axioms was found by Lněnička and Matúš in [17, Lemma 10, (20)]. The offending gaussoid is $\{(12 \mid 3),(13 \mid 4),(14 \mid 2)\}$ over $N=1234$. It contains the antecedents of the instance in dimension 4 of Studený's infinite list of independent inference rules [35]. This family appears in the same function in infinite forbidden minor proofs for semimatroids [20, Proposition 4] and (semidefinite) Gaussian CI structures [30, Theorem 3.2].

In private correspondence, Milan Studený kindly pointed out that Corollary 3.1 is not a complete analogue to [36] because it concerns only CI inference forms over elementary or local CI statements $(i j \mid K) \in \mathcal{A}_{N}$ where $i$ and $j$ are singletons. By contrast, statements of the form $(I, J \mid K)$ with pairwise disjoint sets $I, J, K \subseteq N$ are global CI statements. It is an easy exercise to prove that for compositional graphoids the following equivalence holds:

$$
(I, J \mid K) \Leftrightarrow \bigwedge_{i \in I, j \in J}(i j \mid K) .
$$

Therefore, a general theory of gaussoids can forgo global symbols. Since local and global semigraphoids are in bijection, a global semigraphoid can be recovered exactly from its intersection with $\mathcal{A}_{N}$ [20, Section 2]. However, for inference rules with a bound on the number of antecedents, there is a major difference in allowing global statements. For example, the single global CI statement $(12,34 \mid 5)$ corresponds to the local statements $\{(13 \mid 5),(14 \mid 5),(23 \mid 5),(24 \mid 5)\}$ which have a unique minimal gaussoid extension with 16 elements. This gaussoid is realizable, hence $(12,34 \mid 5)$ is not the antecedent set of a nontrivial valid global inference rule for Gaussians, but this is not covered by our proof. Hence we have

Conjecture 7.1 All minimal gaussoid extensions of at most two global CI statements are realizable.

In 2004, in an effort to classify semigraphoids by closures of families of "easy semigraphoids" under certain operations, Matúš [21] proved a significant refinement of Studený's two-antecedental completeness: his Theorem 2 states that all minimal semigraphoid extensions of two global CI statements are multilinear over every field, which implies discrete realizability and hence, by Lemma 3.1, two-antecedental completeness of the semigraphoid axioms for discrete CI and also for multilinearity over every field. Following [19], a semigraphoid is a semimatroid if it is the CI structure of a polymatroid rank function. It is multilinear (depending on context this is also called just linear) if the rank function is given by a subspace arrangement, cf. [20, Section 6.1]. Multilinearity over every field of all semigraphoids with at most two generators is a strong result which may be helpful in approaching Conjecture 7.1. However, there is no general relation between multilinear 
semimatroids which happen to be gaussoids and algebraic or positive realizability, as the following example shows.

Example 7.1 (A multilinear but non-complex gaussoid) To find a 5-gaussoid which is not algebraic over the complex numbers, one iterates through the $B_{5}$-orbit representatives of 5-gaussoids computed in [3]. Remark 4.1 describes a complex realizability test for gaussoids in geometric terms, which is readily translated into the language of commutative algebra, to be executed by a computer algebra system like Macaulay2 [13]. We refer to [6, Section 4.4] for further clarification of the machinery of computer algebra. However, the exact test from Remark 4.1 requires computing the following quotient of the radical ideal defined by the gaussoid-under-test $\mathcal{M}$ :

$$
\sqrt{\left\langle\operatorname{det} \Gamma_{i j \mid K}:(i j \mid K) \in \mathcal{M}\right\rangle}:\left(\prod_{K \subseteq N} \operatorname{det} \Gamma_{K} \cdot \prod_{(i j \mid K) \notin \mathcal{M}} \operatorname{det} \Gamma_{i j \mid K}\right)
$$

where $\Gamma$ is a generic symmetric $5 \times 5$ matrix over $\mathbb{C}$. The product on the right is a very large polynomial and computing the quotient is often infeasible due to the amount of memory and CPU time required. Instead of taking the ideal quotient by a product of minors, one can successively take the quotient by each factor. This produces a potentially larger variety than the realization space of $\mathcal{M}$ described in Remark 4.1, but the computation is feasible and if the larger variety turns out to be empty, the gaussoid is certain to be non-realizable. For some gaussoids, this method proves non-realizability within a few seconds.

The multilinearity test is based on the characterization of the cone spanned by multilinear polymatroids on five variables achieved by Dougherty-Freiling-Zeger in [8]. We thank Kenneth Zeger for pointing us to the new location of the data mentioned in their paper, http:// code.ucsd.edu/zeger/linrank. Using the list of extreme rays of the multilinear polymatroid cone, one can compute their CI structures and check for every incoming non-algebraic gaussoid from the preceding test whether it is equal to the intersection of all extreme structures above it.

One gaussoid passing both tests is

$$
\mathcal{M}=\{(14 \mid 23),(14 \mid 35),(15 \mid 2),(15 \mid 4),(23 \mid 145),(24 \mid 5),(25 \mid 13),(34 \mid 1),(35 \mid)\}
$$

To confirm that this gaussoid is non-realizable over $\mathbb{C}$, it suffices to write down the ideal defined by the almost-principal minors in $\mathcal{M}$, compute its radical and saturate it at the product of the non-vanishing entries of the matrix as specified by $\mathcal{M}$, which is a fast operation. The variety defined by the resulting ideal is a superset of the algebraic realization space of $\mathcal{M}$ and one can check that the almost-principal minor (34|125) $\notin \mathcal{M}$ vanishes on this variety. This proves the nine-antecedental inference rule

$$
\bigwedge \mathcal{M} \Rightarrow(34 \mid 125) \vee(12 \mid) \vee(13 \mid) \vee(14 \mid) \vee(15 \mid) \vee(23 \mid) \vee(24 \mid) \vee(25 \mid) \vee(34 \mid) \vee(45 \mid)
$$

for algebraic Gaussians over $\mathbb{C}$. Since $\mathcal{M}$ does not satisfy this rule, it is not realizable over $\mathbb{C}$. To prove multilinearity, one has to exhibit the face of the multilinear polymatroid cone defined by $\mathcal{M}$ and verify that a relatively interior point realizes $\mathcal{M}$. This face is spanned by the following 37 extreme rays. Each ray is written as an array of 31 single-digit integers in 
"binary counter" order, i.e., the ranks are listed in the order of 1, 2, 12, 3, 13, 23, 123, 4, ., omitting the empty set at the beginning whose value is always 0 :

\begin{abstract}
$0001100110111110111111111111111 \quad 10100111110011011111111011111111100011111110001111111110111111$ 101101111110111111111111111111111100111111111011111111111111111101111111111111111111111111111 $0011101111112221112222222222222 \quad 1010112121011212122221212222222 \quad 1110122122111212222222212222222$ $1110112122121212122222222222222 \quad 1111021212212112222122222222222 \quad 1210122122221212222222222222222$ $1111122122212222222222222222222 \quad 110112122122112233223223233333311111212222222223322333333333333$ $12111322233222233332333333333331111122222222223333232333333333 \quad 1111122222222222333333332333333$ 112112222222332233333333333333321211232332233232333333333333331111222232232333343343444444444 $1211232233332333443344444444444 \quad 11221223233243334343444444444441121223232233433344443444444444$ 1121223233233433344444444444444222113333433332444434444444444412212332333334334444444444444444 $12222333344344445454555555555552132234344335544455555555555555 \quad 2321244345443435555455555555555$ 323214444543543555545555555555522222444444444455665656656666662232244445445545666566666666666 213233535434565546666566666666622323454554556565776767777777772332355456555656777677777777777 3243255556547656777677777777777
\end{abstract}

The fact that these vectors are multilinear and even extreme rays is the content of [8]. It is easy to verify that the CI structure of the sum of the above vectors is exactly equal to $\mathcal{M}$. In their published data, [8] give integer matrices whose rowspans represent the subspace arrangement. These matrices have the additional property that whenever a concatenation of them has rank $r$ over $\mathbb{Q}$, then there exists an $r \times r$ submatrix whose determinant equals \pm 1 , thus proving that the matrices realize the same polymatroid over every field. By the general theory of multilinear semimatroids, this shows that $\mathcal{M}$ is multilinear over every field as well. The process and code snippets are more thoroughly documented at https://github. com/taboege/gaussant-code.

Putting together results by Matúš-Studený [23-25] (corrections by Šimeček [32]) and Lněnička-Matúš [17], one finds that on the set of gaussoids on a four-element ground set, the realizability by discrete random variables and by positive-definite matrices over $\mathbb{Q}$ or $\mathbb{R}$ are equivalent. In particular, a ground set of cardinality five is required to find a multilinear but non-algebraic gaussoid.

The notion of realizability near a matrix which is in the hyperoctahedral orbit of the identity matrix was crucial to the proof of the main theorem in Section 6. We showed that all gaussoids generated from at most two CI statements are rationally realizable near the identity matrix. In the following we present counterexamples to some obvious questions about near-identity realizability.

Example 7.2 (A non-near-identity realizable gaussoid) Entry № 20 in [17, Table 1] contains the curve of matrices

$$
\left(\begin{array}{llll}
1 & 2-\delta^{-2} & \delta & \delta \\
2-\delta^{-2} & 1 & 0 & \delta \\
\delta & 0 & 1 & \delta^{2} \\
\delta & \delta & \delta^{2} & 1
\end{array}\right) \longrightarrow\left(\begin{array}{cccc}
1 & \frac{2}{9} & \frac{3}{4} & \frac{3}{4} \\
\frac{2}{9} & 1 & 0 & \frac{3}{4} \\
\frac{3}{4} & 0 & 1 & \frac{9}{16} \\
\frac{3}{4} & \frac{3}{4} & \frac{9}{16} & 1
\end{array}\right), \text { as } \delta \rightarrow \frac{3}{4}
$$

The gaussoid $\mathcal{G}=\{(13 \mid 24),(23 \mid),(34 \mid 1)\}$ realized by this matrix is the algebraic Gaussian in our sense over $\mathbb{Q}(\varepsilon)$ where $\frac{3}{4}+\varepsilon$ is substituted for $\delta$. 
Consider the slice of the positive realization space over $\mathbb{R}$ of this gaussoid on the affinelinear space of symmetric matrices

$$
\Sigma=\left(\begin{array}{llll}
1 & a & b & c \\
a & 1 & 0 & e \\
b & 0 & 1 & f \\
c & e & f & 1
\end{array}\right) .
$$

This slice is the intersection of the algebraic realization space of the gaussoid with the elliptope. The identity matrix lies in the center of the elliptope and we wish to show that the realization space of $\mathcal{G}$, although non-empty, does not approach this center. In particular $\mathcal{G}$ is a gaussoid with three elements, rationally positively realizable, but not realizable near the identity. On the given slice, these two equations hold:

$$
\begin{gathered}
f=b c, \\
b+a e f=c f+b e^{2} .
\end{gathered}
$$

Substituting the first into the second equality and canceling the non-zero factor $b$ in every term we find

$$
1+\text { ace }=c^{2}+e^{2} \text {. }
$$

This equation cannot be satisfied if $a, c$ and $e$ all tend to zero. It can be shown that the realization space of $\mathcal{G}$ decomposes into eight reorientation classes which are identical up to an orthogonal transformation; for an explanation of reorientation, see [3, Section 5]. This transformation preserves Euclidean distances and it fixes the center of the elliptope, thus all of these components have the same distance to the identity matrix. Focusing on one of them, we can assume that $a, c$ and $e$ are all positive and then the Euclidean distance of a realization of $\mathcal{G}$ to the identity is

$$
\sqrt{2} \sqrt{a^{2}+b^{2}+c^{2}+e^{2}+f^{2}}=\sqrt{2} \sqrt{1+a c e+a^{2}+b^{2}+f^{2}} \geq \sqrt{2} .
$$

By allowing $e$ to converge to one while $a, b$ and $c$ converge to zero, one can find positivedefinite realizations of $\mathcal{G}$ which approach this lower bound. Thus, the elliptope slice of the realization space of $\mathcal{G}$ has distance $\sqrt{2}$ to the identity matrix.

It was remarked in [3, Corollary 1] that, based on the hyperoctahedral action and [17, Table 1], every 4-gaussoid is algebraically realizable over $\mathbb{C}$. Since all realizations in the table are even rational, our Lemma 5.1 and Proposition 4.1 furthermore imply that every 4-gaussoid is algebraically realizable over $\mathbb{Q}$, while not all of them are positively realizable even over $\mathbb{R}$.

Example 7.3 (Near-identity realizability not preserved under $B_{N}$ ) The bracketed selfdual gaussoid $\{(12 \mid),(12 \mid 34),(34 \mid 1),(34 \mid 2)\}$ in item $4_{12}$ in [3, p. 15] is not positively realizable over $\mathbb{R}$. However, in its hyperoctahedral orbit is the likewise self-dual $\{(12 \mid 3),(12 \mid 4),(34 \mid 1),(34 \mid 2)\}$ and this gaussoid is even realizable rationally near the identity matrix — it is № 30 in [17, Table 1].

Example 7.4 (A non-algebraic gaussoid) In [3, Example 13] a 5-gaussoid was found which is not algebraically realizable over $\mathbb{C}$. This gaussoid had a redundant element (25|34) which contributed neither to the property of being a gaussoid nor to being non-realizable. In this 
example, we consider the gaussoid of [3, Example 13], with (25|34) removed, from the broader perspective of algebraic realizability over general fields. The claim is that

$$
\mathcal{V}=\{(12 \mid),(13 \mid 4),(14 \mid 5),(23 \mid 5),(35 \mid 1),(45 \mid 2),(15 \mid 23),(34 \mid 12),(24 \mid 135)\}
$$

is not algebraically realizable over any field. It is sufficient to check this over algebraically closed fields. Over these fields, we can impose a unit diagonal on a principally regular realization $\Gamma$, by Remark 4.2. Then, $\mathcal{V}$ imposes the following easy equations on $\Gamma$ :

$$
\Gamma=\left(\begin{array}{lllll}
1 & 0 & b & c & d \\
0 & 1 & e & f & g \\
b & e & 1 & h & i \\
c & f & h & 1 & j \\
d & g & i & j & 1
\end{array}\right), \quad e=g i, \quad \begin{aligned}
& i=b d, \quad b=d j, \\
& b=c h, \quad j=f g .
\end{aligned}
$$

Using these variable substitutions, the longer equations, corresponding to CI statements with bigger conditioning sets, shrink. They are

$$
\begin{gathered}
0=d\left[1-d^{2} f^{2} g^{2} h^{2}\left(1+d^{2} g^{2}-g^{2}\right)\right], \\
0=h\left[1-2 d^{2} f^{2} g^{2}\right], \\
0=f\left[\left(1-d^{2}\right)\left(1+d^{2} f^{2} g^{4} h^{2}\left(1+d^{2}\right)-g^{2}\left(1+d^{2} h^{2}\left(1+f^{2}\right)\right)\right)\right] .
\end{gathered}
$$

Dividing by the non-zero variables $d, f$, and $h$ yields a system in even powers of the variables. Replacing $d^{2}=D$ and so on, we have:

$$
1=D F G H(1+D G-G),
$$

$$
1=2 D F G
$$

$$
0=(1-D)\left(1+D F G^{2} H+D^{2} F G^{2} H-G-D G H-D F G H\right) .
$$

In a principally regular $\Gamma$ we have $1-D=\operatorname{det} \Gamma_{15} \neq 0$, so the last equation is equivalent to

$$
0=1+D F G^{2} H+D^{2} F G^{2} H-G-D G H-D F G H .
$$

By adding up (a) and $\left(\mathrm{c}^{\prime}\right)$ and then using (b):

$$
\begin{aligned}
0= & 1+D F G^{2} H+D^{2} F G^{2} H-G-D G H-D F G H+ \\
& \quad D F G H+D^{2} F G^{2} H-D F G^{2} H-1 \\
= & 2 D^{2} F G^{2} H-D G H-G \\
= & -G,
\end{aligned}
$$

which is a contradiction to $(25 \mid) \notin \mathcal{V}$. Notice that this contradiction was derived using only the non-vanishing of principal minors and division by variables known to be non-zero by the definition of $\mathcal{V}$. The argument is independent of the field chosen, so the following nine-antecedental inference rule is valid for algebraic Gaussians over every field:

$$
\bigwedge \mathcal{V} \Rightarrow(15 \mid) \vee(24 \mid) \vee(25 \mid) \vee(34 \mid)
$$

This proves that $\mathcal{V}$ is not algebraically realizable over any field. 
We note that, unlike $\mathcal{M}$ of Example 7.1, this CI structure is not realizable by discrete random variables either because it is not a semimatroid. The closure of $\mathcal{V}$ in the set of 5 -semimatroids and in the set of multilinear 5-semimatroids can be computed using polyhedral geometry, as outlined in Example 7.1, because the extreme rays of the relevant cones are known. The semimatroid closure of $\mathcal{V}$ contains $16 \mathrm{CI}$ statements but none of the conclusions (15|), (24|), (25|) and (34|) of (†), so this inference rule is not valid for semimatroids. The multilinear closure is larger at 22 elements and from the possible conclusions of ( $\$)$, it contains precisely $(15 \mid)$. Hence, the special case $\wedge \mathcal{V} \Rightarrow(15 \mid)$ of (†) is valid for multilinear semimatroids. The closure of $\mathcal{V}$ among the $\mathrm{CI}$ structures realizable by discrete random variables lies in between the semimatroid and multilinear closures. It remains open whether $\bigwedge \mathcal{V} \Rightarrow(15 \mid)$ is valid even in the discrete case.

As a final direction for future work we would like to mention the influence of the field $\mathbb{K}$ on the realizability of gaussoids. There are numerous questions to be asked inspired by related theorems in matroid theory, cf. [27, Chapter 6]. To give one example: recall from Remark 4.1 that the realizability of a gaussoid $\mathcal{G}$ is a disproof of the existence of a non-trivial valid inference rule with antecedent set $\mathcal{G}$. Therefore, realizing matrices represent invalidity proofs for inference rules in a very compact way. In the proof of Theorem 3.1, we exhibited rational matrices as invalidity proofs for all two-antecedental inference formulas which are not implied by the gaussoid axioms. These matrices can be stored on a computer and verified using exact arbitrary-precision rational arithmetic. This leads to the following two variants of a question which was asked before by Petr Šimeček [31, Section 4] in the broader context of semidefinite Gaussian CI models. He asked whether there exists a realizable Gaussian CI structure for which a rational realizing covariance matrix does not exist - because his computer search for invalid inference rules was conducted on rational matrices only. Both questions are answered affirmatively in [2].

\section{Question 7.1 Is there a gaussoid which is positively realizable over $\mathbb{R}$ but not over $\mathbb{Q}$ ?}

\section{Question 7.2 Are there gaussoids which separate the notions of algebraic realizability over} $\mathbb{C}, \mathbb{R}$ and $\mathbb{Q}$ ?

Acknowledgements The author wishes to thank Thomas Kahle, Andreas Kretschmer and Milan Studený for helpful discussions, and the anonymous referees for their clear and thoughtful suggestions for improvement of the earlier manuscript. Thanks to Xiangying Chen for spotting an error in the previous version of Example 7.4 .

Funding Open Access funding enabled and organized by Projekt DEAL. This work is funded by the Deutsche Forschungsgemeinschaft (DFG, German Research Foundation) - 314838170, GRK 2297 MathCoRe.

\section{Declarations}

Conflict of Interests The author declares that he has no conflict of interest.

Open Access This article is licensed under a Creative Commons Attribution 4.0 International License, which permits use, sharing, adaptation, distribution and reproduction in any medium or format, as long as you give appropriate credit to the original author(s) and the source, provide a link to the Creative Commons licence, and indicate if changes were made. The images or other third party material in this article are included in the article's Creative Commons licence, unless indicated otherwise in a credit line to the material. If material is 
not included in the article's Creative Commons licence and your intended use is not permitted by statutory regulation or exceeds the permitted use, you will need to obtain permission directly from the copyright holder. To view a copy of this licence, visit http://creativecommons.org/licenses/by/4.0/.

\section{References}

1. Abo Khamis, M., Kolaitis, P.G., Ngo, H.Q., Suciu, D.: Decision problems in information theory. arXiv:2004.08783 (2020)

2. Boege, T.: Incidence geometry in the projective plane via almost-principal minors of symmetric matrices. arXiv:2103.02589 (2021)

3. Boege, T., D’Alì, A., Kahle, T., Sturmfels, B.: The geometry of gaussoids. Found. Comput. Math. 19(4), 775-812 (2019). https://doi.org/10.1007/s10208-018-9396-X

4. Boege, T., Kahle, T.: Construction methods for gaussoids. Kybernetika 56(6), 1045-1062 (2020). https://doi.org/10.14736/kyb-2020-6-1045

5. Bouckaert, R., Hemmecke, R., Lindner, S., Studený, M.: Efficient algorithms for conditional independence inference. J. Mach. Learn. Res. 11, 3453-3479 (2010)

6. Cox, D.A., Little, J., O'Shea, D.: Ideals, varieties, and algorithms, fourth edn. Undergraduate Texts in Mathematics. Springer, Berlin (2015). https://doi.org/10.1007/978-3-319-16721-3. An introduction to computational algebraic geometry and commutative algebra

7. Dawid, A.P.: Conditional independence in statistical theory. J. Roy. Statist. Soc. Ser. B 41(1), 1-31 (1979)

8. Dougherty, R., Freiling, C., Zeger, K.: Linear rank inequalities on five or more variables. arXiv:0910.0284 (2010)

9. Drton, M., Xiao, H.: Smoothness of gaussian conditional independence models. In: Algebraic Methods in Statistics and Probability II, Contemporary Mathematics, vol. 516, pp. 155-177. American Mathematical Society (2010). https://doi.org/10.1090/conm/516/10173

10. Geiger, D., Pearl, J.: Logical and algorithmic properties of conditional independence and graphical models. Ann. Stat. 21(4), 2001-2021 (1993). https://doi.org/10.1214/aos/1176349407

11. Geiger, D., Pearl, J.: Logical and algorithmic properties of conditional independence and graphical models. Ann. Statist. 21(4), 2001-2021 (1993). https://doi.org/10.1214/aos/1176349407

12. Gong, Z., Aldeen, M., Elsner, L.: A note on a generalized Cramer's rule. Linear Algebra Appl. 340(1), 253-254 (2002)

13. Grayson, D.R., Stillman, M.E.: Macaulay2, a software system for research in algebraic geometry. Available at http://www.math.uiuc.edu/Macaulay2/

14. Holtz, O., Sturmfels, B.: Hyperdeterminantal relations among symmetric principal minors. J. Algebra 316(2). https://doi.org/10.1016/j.jalgebra.2007.01.039 (2007)

15. Lauritzen, S.: Graphical models., Oxford Statistical Science series, vol. 17. Oxford University Press, Oxford (1996)

16. Lauritzen, S., Sadeghi, K.: Unifying Markov properties for graphical models. Ann. Statist. 46(5), 22512278 (2018). https://doi.org/10.1214/17-AOS1618

17. Lněnička, R., Matúš, F.: On Gaussian conditional independence structures. Kybernetika 43(3), 327-342 (2007)

18. Marshall, M.: Positive polynomials and sums of squares, Mathematical Surveys and monographs, vol. 146 American Mathematical Society (2008)

19. Matúš, F.: Probabilistic conditional independence structures and matroid theory: background. Int. J. Gen. Syst. 22, 185-196 (1994)

20. Matúš, F.: Conditional independence structures examined via minors. Ann. Math. Artif. Intell. 21(1), 99-30 (1997). https://doi.org/10.1023/A:1018957117081

21. Matúš, F.: Towards classification of semigraphoids. Discrete Math. 277(1), 115-145 (2004). https://doi.org/10.1016/S0012-365X(03)00155-9

22. Matúš, F.: Conditional independences in gaussian vectors and rings of polynomials. In: Kern-Isberner, G., Rödder, W., Kulmann, F. (eds.) Conditionals, Information, and Inference, pp. 152-161. Springer (2005)

23. Matúš, F.: Conditional independences among four random variables. II. Combin. Probab. Comput. 4(4), 407-417 (1995). https://doi.org/10.1017/S0963548300001747

24. Matúš, F.: Conditional independences among four random variables. III. Final conclusion. Combin. Probab. Comput. 8(3), 269-276 (1999). https://doi.org/10.1017/S0963548399003740 
25. Matúš, F., Studený, M.: Conditional independences among four random variables. I. Combin. Probab. Comput. 4(3), 269-278 (1995). https://doi.org/10.1017/S0963548300001644

26. Niepert, M., Gyssens, M., Sayrafi, B., Van Gucht, D.: On the conditional independence implication problem: a lattice-theoretic approach. Artif. Intell. 202, 29-51 (2013). https://doi.org/10.1016/j.artint.2013. 06.005

27. Oxley, J. Matroid theory, Oxford Graduate Texts in Mathematics, 2nd edn., vol. 21. Oxford University Press, Oxford (2011). https://doi.org/10.1093/acprof:oso/9780198566946.001.0001

28. Pearl, J.: Probabilistic reasoning in intelligent systems: networks of plausible inference. The Morgan Kaufmann Series in Representation and Reasoning. Morgan Kaufmann, San Mateo CA (1988)

29. Pearl, J., Paz, A.: GRAPHOIDS: A Graph-based logic for reasoning about relevance relations, or When would $x$ tell you more about y if you already know z. Tech. Rep. CSD-850038 UCLA Computer Science Department (1985)

30. Šimeček, P.: Classes of Gaussian, discrete and binary representable independence models have no finite characterization. In: Proceedings of Prague Stochastics, vol. 400, pp. 622-631 (2006)

31. Šimeček, P.: Gaussian representation of independence models over four random variables. In: COMPSTAT Conference (2006)

32. Šimeček, P.: A short note on discrete representability of independence models. In: Proccedings of the European Workshop on Probabilistic Graphical Models, pp. 287-292 (2006)

33. Studený, M.: Probabilistic conditional independence structures. Information science and statistics springer (2005)

34. Studený, M.: Conditional independence and basic markov properties. In: Maathuis, M., Drton, M., Lauritzen, S., Wainwright, M. (eds.) Handbook of graphical models, Chapman \& Hall/CRC Handbooks of Modern Statistical Methods, pp. 3-38. CRC Press (2019)

35. Studený, M.: Conditional independence relations have no finite complete characterization. In: Information Theory, Statistical Decision Functions and Random Processes, Vol. B, pp. 377-396. Kluwer (1992)

36. Studený, M.: Semigraphoids are two-antecedental approximations of stochastic conditional independence models. In: De Mantaras, R.L., Poole, D., Proceedings, U.ncertainty. (eds.), pp. 546-552, Morgan Kaufmann (1994)

37. Sullivant, S.: Gaussian conditional independence relations have no finite complete characterization. J. Pure Appl. Algebra 213(8), 1502-1506 (2009)

38. Sullivant, S.: Algebraic statistics, Graduate Studies in Mathematics, vol. 194 American Mathematical Society (2018)

39. Verma, T., Pearl, J.: An algorithm for deciding if a set of observed independencies has a causal explanation. In: Dubois, D., Wellman, M.P., D’Ambrosio, B., Smets, P. (eds.) Uncertainty in Artificial Intelligence, pp. 323-330, Morgan Kaufmann (1992). https://doi.org/10.1016/B978-1-4832-8287-9.50049-9

Publisher's note Springer Nature remains neutral with regard to jurisdictional claims in published maps and institutional affiliations. 